\title{
二维材料的拉曼光谱研究进展
}

\author{
吴娟霞 $1,2,3$, 谢黎明 $1,2,3^{*}$
}

1. 国家纳米科学中心, 中国科学院纳米标准与检测重点实验室, 北京 100190 ;

2. 国家纳米科学中心, 中国科学院纳米科学卓越创新中心, 北京 100190;

3. 中国科学院大学纳米科学与技术学院, 国际学院, 北京 100049

* 联系人, E-mail: xielm@nanoctr.cn

2018-08-15 收稿, 2018-09-06 修回, 2018-09-10 接受, 2018-10-19 网络版发表 中国科学院前沿科学重点研究项目(QYZDB-SSW-SYS031)、中国科学院战略性先导科技专项(B类)(XDB30000000)、国家自然科学基金 (21673058)和北京市人才计划(2015000021223ZK17)资助

摘要 二维材料因其独特的结构与性质引起了科学家们的广泛关注. 拉曼光谱是一种特征性强、快速、无损的 材料结构表征方法, 其在低维材料的结构表征方面具有独特的优势. 本文主要综述了拉曼光谱在二维材料结构表 征方面的研究进展。首先，系统介绍了二维材料结构和拉曼选律基础知识，并分析了二维材料的典型拉曼特征; 其次, 通过对二维材料的典型拉曼特征峰的峰位和峰强的分析, 讨论了拉曼光谱测定二维材料的层数、边缘手性/ 晶格取向、合金成分等; 然后, 介绍了缺陷、掺杂、外界应力以及热效应对二维材料拉曼散射的影响; 最后, 结合 二维电荷密度波材料相变过程中的结构和拉曼特征的变化, 讨论了拉曼光谱在相变性质研究中的应用.

关键词二维材料, 拉曼光谱, 结构表征, 拉曼选律

自2004年英国曼彻斯特大学的Novoselov等人 ${ }^{[1]}$ 首次使用机械剥离获得单层石墨烯以来, 科学家们 对二维材料的研究翻开了新篇章. 石墨烯的原子级 厚度特性赋予了其独特的电子结构和优异的物理化 学性质, 如无质量狄拉克费米子 ${ }^{2]}$ 、整数/分数量子霍 尔效应 ${ }^{[3,4]}$ 、精细常数决定的透光率 $(97.7 \%)^{[5]}$ 、超高 热导率 $\left(5000 \mathrm{~W} \mathrm{~m}^{-1} \mathrm{~K}^{-1}\right)^{[6]}$ 、超高迁移率 $\left(>10^{5} \mathrm{~cm}^{2} \mathrm{~V}^{-1}\right.$

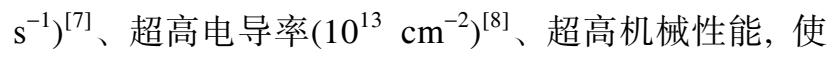
其在纳电子学器件 ${ }^{[9]}$ 、柔性电子器件 ${ }^{[10]}$ 、集成电路以 及光电检测 ${ }^{[11]}$ 等领域具有潜在应用.

然而, 石墨烯零带隙的特性使得其无法兼顾高 迁移率和高的开关比, 科学家们通过垂直施加栅 压 $^{[12]}$ 、不对称双面修饰 ${ }^{[13]}$ 、氮或硼掺杂 ${ }^{[14]}$ 、氢化 ${ }^{[15]}$ 、 氯化 ${ }^{[16]}$ 、氟化 ${ }^{[17]}$ 等手段来打开石墨烯的带隙, 来进一 步扩展其应用领域. 同时, 半导体性二维材料因具有
高的开关比 ${ }^{[18]}$ 、强的光吸收 ${ }^{[19]}$ 等特点, 进入了科学家 们的视线. 短短 10 余年时间内, 二维材料家族得到了 极大的充实 ${ }^{[20]}$ (图1), 包括六方蜂窝状结构的石墨烯 和六方氮化嗍 ${ }^{[21]}$ 、三明治结构的过渡金属二硫族化 合物(如 $\mathrm{MoS}_{2}{ }^{[18]}, \mathrm{MoSe}_{2}{ }^{[22]}, \mathrm{WS}_{2}{ }^{[23]}, \mathrm{WSe}_{2}{ }^{[24]}, \mathrm{ZrS}_{2}{ }^{[25]}$, $\mathrm{TaS}_{2}{ }^{[26]}, \mathrm{ReS}_{2}{ }^{[27]}$ 等)、第三主族硫化物 (如 $\mathrm{GaSe}^{[28]}$, $\mathrm{GaTe}^{[29]}$ )、第四主族硫化物 (如 $\mathrm{SnSe}^{[30]}, \mathrm{SnS}_{2}{ }^{[31]}$, $\left.\mathrm{SnSe}_{2}{ }^{[32]}\right)$ 、黑磷 ${ }^{[33]}$ 以及硅烯 ${ }^{[34]}$ 、锗烯 ${ }^{[35]}$ 等. 其中对过 渡金属二硫族化合物 $\left(\mathrm{MX}_{2}\right)^{[36,37]}$ 的研究最为广泛, 其 金属原子 $\mathrm{M}$ 主要分布在第四副族 $(\mathrm{Ti}, \mathrm{Zr}, \mathrm{Hf})$ 、第五副 族 $(\mathrm{V}, \mathrm{Nb}, \mathrm{Ta}) 、$ 第六副族 $(\mathrm{Mo}, \mathrm{W})$ 和第七副族 $(\mathrm{Re})$, 随 着金属原子或者硫族原子的不同, $\mathrm{MX}_{2}$ 表现出丰富的 能带结构, 其物理化学性质也各有不同 ${ }^{[36]}$, 如半导 体性的 $\mathrm{MoS}_{2}$ 和 $\mathrm{WSe}_{2}$ 、半金属性的 $\mathrm{WTe}_{2}$ 和 $\mathrm{TiSe}_{2}$ 、以及 金属性的 $\mathrm{NbS}_{2}$ 和 $\mathrm{TaS}_{2}$. 二维材料可表现出与其块体 


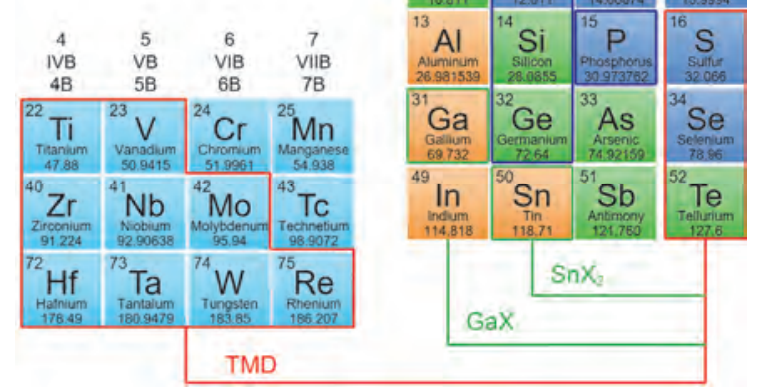

图 1 常见二维材料在元素周期表中的分布 ${ }^{[20]}$

Figure 1 Distribution of two-dimensional (2D) materials in periodic table ${ }^{[20]}$

材料截然不同的性质, 比如较大的比表面积、独特的 电子能带结构 ${ }^{[2,38,39]}$ 、表面无悬挂件 ${ }^{[18]}$ 、柔性、超轻 等. 如随着层数减少, $\mathrm{MoS}_{2}$ 和 $\mathrm{WS}_{2}$ 等出现间接带隙向 直接带隙的转变 ${ }^{[40]}$.

二维材料由于其优异的性质成为材料学领域研 究中的佼佼者, 那么对其结构进行精确、快速表征显 得非常重要. 光学显微镜 $(\mathrm{OM})^{[41,42]}$ 、扫描探针显微镜 $(\mathrm{SPM})^{[43]}$ 、扫描电子显微镜 $(\mathrm{SEM})$ 、透射电子显微镜 $(\mathrm{TEM})^{[44]}$ 以及拉曼光谱等技术均被用来表征二维材 料的结构. 石墨烯在2004年被发现时就是通过OM观 察到的 ${ }^{[1]}$, 这主要是基于光的干涉作用导致样品和基 底区域形成高的衬度差 ${ }^{[42,45]}$, 这一方法可以对二维 材料的厚度和形貌进行快速地表征, 但是其特征性 差, 且需要固定介电层厚度、光波长等参数; 与 $\mathrm{OM}$ 相比, SPM和TEM可以以更高的分辨率对二维材料的 层数、层间堆垛方式以及表面形貌进行表征, 但是其 表征范围小, 速度较慢, 制样要求较高. 如, STM和 TEM均需要超高真空的环境, STM还需要样品在导 电基底上, 因此其在二维材料的快速表征方面受到 限制.

拉曼光谱是一种表征材料的晶体结构、电子能带 结构、声子色散以及电子-声子相互作用的技术手段, 其具有快速、便捷、无损、高空间分辨率等特点, 在 二维材料的发展历程中起到了至关重要的作用. 拉 曼光谱在二维材料的结构表征中具有独特的优势,

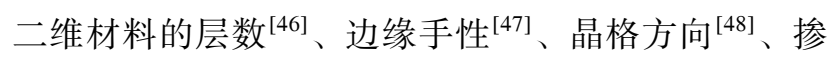
杂 ${ }^{[49,50]}$ 、合金成分 ${ }^{[11]}$ 、所受应力 ${ }^{[52]}$ 、热效应 ${ }^{[53]}$ 以及
结构相变 ${ }^{[54]}$ 均可以在其拉曼散射(峰位、峰强以及半 峰宽等)中体现. 通过对拉曼散射峰进行分析, 不仅 可以对二维材料的这些结构参数和一些物理参量进 行比较准确的测定, 还可以对其拉曼散射过程中电 子-声子耦合等一些物理过程进行系统研究.

本文主要综述了拉曼光谱技术在二维材料结构 表征方面的研究进展, 主要包括: (1) 根据二维材料 的结构对称性和拉曼散射强度的计算方法, 介绍了 常见的二维材料的典型拉曼特征模; (2) 讨论了二维 材料的典型拉曼特征峰的强度和峰位对其层数、边缘 手性、晶格取向和合金成分的指认/测量; (3) 结合新 峰的出现以及其与二维材料典型拉曼特征峰的强度 比, 介绍了拉曼光谱对二维材料缺陷密度的测定; (4) 介绍了掺杂、外界应力以及热效应对二维材料拉曼特 征峰的影响, 据此讨论了拉曼光谱对二维材料掺杂 浓度、应力以及热导率的测定；(5) 介绍并分析了拉 曼光谱在二维电荷密度波材料相变特性研究中的 应用.

\section{1 二维材料的拉曼光谱}

单层石墨烯是由 $\mathrm{sp}^{2}$ 碳原子紧密堆积形成的具有 六方蜂窝状结构的二维平面结构 ${ }^{[5]}$. 单层六方氮化 嗍与石墨烯结构类似, 只是用 $\mathrm{B}$ 和 $\mathrm{N}$ 原子分别替代了 石墨烯晶胞中的 $\mathrm{A}, \mathrm{B}$ 碳原子, 因此以三重旋转轴替 代了石墨烯的六重旋转轴 ${ }^{[56]}$. 过渡金属二硫族化合 物 $\left(\mathrm{MX}_{2}\right)$ 具有类似于石墨烯的层状结构, 其每一层都 是由一层金属原子填充在两层硫族原子中间形成 $\mathrm{X}-\mathrm{M}-\mathrm{X}$ 的三明治结构, 层内以 $\mathrm{M}-\mathrm{X}$ 共价键相结合, 而层与层之间以范德华力相互耦合. 如图2(a)所示, 单层 $\mathrm{MX}_{2}$ 的金属原子可以形成三棱柱形或八面体形 的配位结构 ${ }^{[36,57]}$, 不同配位又有不同的层层堆垛结 构, 使得 $\mathrm{MX}_{2}$ 表现出更加丰富的晶体结构, 常见的有 3 种晶型 $2 \mathrm{H}, 3 \mathrm{R}$ 和 $1 \mathrm{~T}$, 其对称性分别为六方、斜方六 面体和三方, 前面的数字代表每一个重复单元所包 含的层数.

晶体结构决定拉曼散射性质, 不同晶体结构所 对应的单胞基矢不同, 从而决定了其所属点群. 属于 相同点群的材料其拉曼张量一样. 以 $\mathrm{MX}_{2}$ 为例, $1 \mathrm{~T}$, $2 \mathrm{H}$ 和 $3 \mathrm{R}$ 晶型的 $\mathrm{MX}_{2}$ 分别属于 $\mathrm{D}_{3 \mathrm{~d}}{ }^{3}, \mathrm{D}_{3 \mathrm{~h}}{ }^{1}$ 和 $\mathrm{D}_{3 \mathrm{v}}{ }^{1}$ 点群 ${ }^{[59]}$. 这里需要说明的是, $2 \mathrm{H}$ 和 $3 \mathrm{R}$ 晶型的 $\mathrm{MX}_{2}$ 均为三棱柱 形的金属配位，其单层结构单元是一致的，表示为 $1 \mathrm{H}$, 对于硫族原子为 $\mathrm{Te}$ 的 $\mathrm{MX}_{2}$ (如 $\mathrm{WTe}_{2}$ ), $\mathrm{Te}$ 原子的尺 

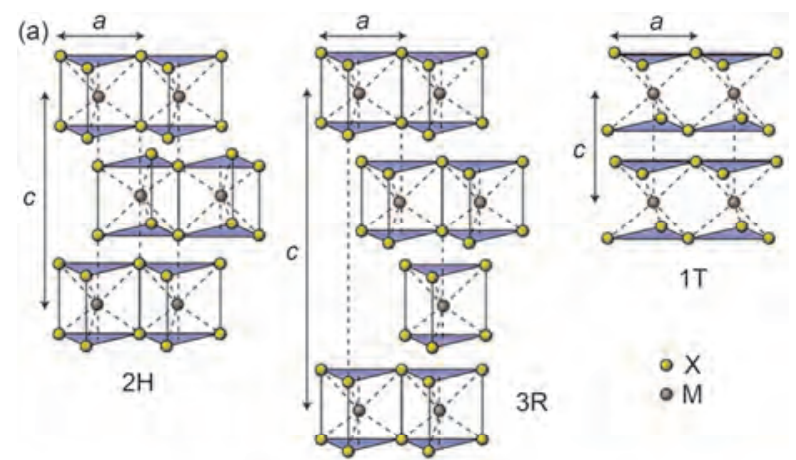

$1 \mathrm{~T}$

$\circ \mathrm{X}$

$\circ \mathrm{M}$
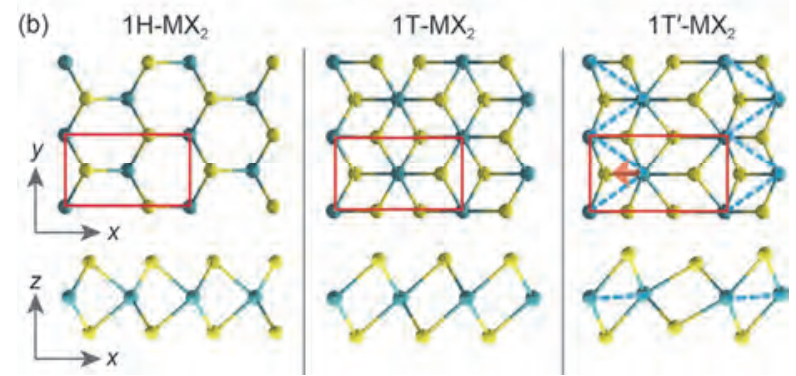

图 $2 \mathrm{MX}_{2}$ 的结构多样性. (a) $2 \mathrm{H}, 3 \mathrm{R}$ 和 $1 \mathrm{~T}$ 晶型的 $\mathrm{MX}_{2}$ 结构示意图 ${ }^{[57]}$; (b) 单层 $\mathrm{MX}_{2}$ 的原子结构的顶视图和侧视图 ${ }^{[58]}$

Figure 2 Structural polytypism in $\mathrm{MX}_{2}$. (a) Sturctural schematics of $2 \mathrm{H}, 3 \mathrm{R}$ and $1 \mathrm{~T} \mathrm{MX}_{2}{ }^{[57]}$; (b) top and side views of atomistic structures of monolayer $\mathrm{MX}_{2}{ }^{[58]}$

寸较大, 会造成结构扭曲, 形成 $1 \mathrm{~T}^{\prime}$ 相, 其单层原子 结构的顶视图和侧视图如图2(b)所示 ${ }^{[58]}$.

在实际的拉曼测量中, 某一振动模的拉曼散射 强度 $S$ 与其拉曼张量 $\tilde{R}$ 的形式和拉曼仪器的光学配置 密切相关, 可以表示为

$$
S \propto\left|e_{\mathrm{i}} \cdot \tilde{R} \cdot e_{\mathrm{s}}\right|^{2}=\left|\left(\begin{array}{lll}
x & y & z
\end{array}\right)\left(\begin{array}{ccc}
\alpha_{x x} & \alpha_{x y} & \alpha_{x z} \\
\alpha_{y x} & \alpha_{y y} & \alpha_{y z} \\
\alpha_{z x} & \alpha_{z y} & \alpha_{z z}
\end{array}\right)\left(\begin{array}{l}
x \\
y \\
z
\end{array}\right)\right|^{2},
$$

其中 $e_{\mathrm{i}}$ 和 $e_{\mathrm{s}}$ 分别为人射光和散射光的偏振矢量. 对于 二维材料来说, 在拉曼光谱的测量中通常是在平行 偏振 $Z(X X) \bar{Z}$ 和交叉偏振 $Z(X Y) \bar{Z}$ 的仪器配置下进 行, 这里 $X$ 轴和 $Y$ 轴与二维材料平面平行, 对应人射 光和散射光的偏振方向, $Z$ 轴平行于人射光和散射光 的传播方向, 在后面的内容中, 仪器的偏振配置仅用 括号中的两个字母表示, 即 $X X$ 为人射光和散射光偏 振方向均沿着 $X$ 方向的平行偏振. 式(1)即为材料的拉 曼选律, 只有当拉曼散射强度不为零时, 这一拉曼振 动模才是具有拉曼活性的, 反之即为拉曼非活性振 动模. 从式(1)可知, 某一振动模在平行偏振下的拉 曼散射强度仅与其拉曼张量的对角矩阵元相关, 而
在交叉偏振下，其拉曼散射强度仅与拉曼张量的非 对角矩阵元相关. 常见二维材料的带隙、所属点群、 布里渊区中心的不可约表示以及典型拉曼特征如表 1 所示. 对于各向同性的二维材料(如 $2 \mathrm{H}-\mathrm{MoS}_{2}, 2 \mathrm{H}-$ $\mathrm{WSe}_{2}$ ), 可以通过偏振拉曼散射强度的计算对其拉曼 特征峰进行归属, 比如 $2 \mathrm{H}-\mathrm{MX}_{2}(\mathrm{M}=\mathrm{Mo}, \mathrm{W} ; \mathrm{X}=\mathrm{S}, \mathrm{Se})$ 的 $\mathrm{A}_{1 \mathrm{~g}}$ 振动模(在奇数层中表现为 $\mathrm{A}^{\prime}{ }_{1}$, 在偶数层中表 现为 $\mathrm{A}_{1 \mathrm{~g}}$ ) 仅可以在平行偏振下检测到, 而 $\mathrm{E}_{2 \mathrm{~g}}{ }^{1}$ 模(在奇 数层和偶数层中分别表现为 $\mathrm{E}^{\prime}$ 和 $\mathrm{E}_{\mathrm{g}}$ ) 在平行偏振和交 叉偏振下均可以检测到, 而对于各向异性的二维材 料(如 $\mathrm{ReS}_{2}$ 、黑磷), 偏振拉曼则可以用来对其晶格方 向进行指认.

\section{2 拉曼光谱法测定二维材料的层数}

由于限域效应, 二维材料的光学性质强烈依赖 于其层数. 拉曼光谱也不例外, 随着层数的变化, 其 拉曼谱峰的峰位、峰强以及峰形均会相应地发生变 化, 使得拉曼光谱成为快速鉴定二维材料层数的一 种有效手段.

拉曼光谱测定石墨烯的层数主要是通过其特征 $G$ 峰的强度、 $G$ 和 $G^{\prime}$ 峰(又称 $2 D$ 峰)的强度比以及 $G^{\prime}$ 峰 的峰形实现的. 首先, 在石墨烯 $/ \mathrm{SiO}_{2} / \mathrm{Si}$ 三层体系中, 考虑人射光的多级干涉和散射光的多级反射的情况 下, 石墨烯的 $\mathrm{G}$ 峰强度在 10 层以内线性增加, 之后会 随着层数的增加而减弱 ${ }^{[95]}$; 其次, 单层石墨烯的 $\mathrm{G}^{\prime}$ 峰强度大于 $\mathrm{G}$ 峰 $^{[46]}$, 且随着层数的增加, $\mathrm{G}$ 峰频率向 低波数位移, 与层数的倒数成线性关系 ${ }^{\left[{ }^{[9]}\right.}$; 最后, 单 层石墨烯的 $\mathrm{G}^{\prime}$ 峰具有完美的洛伦兹峰形, 随着石墨 烯层数增加, 其电子能带结构发生裂分, 双(三)层石 墨烯的 $\mathrm{G}^{\prime}$ 峰可以用4(6)个洛伦兹峰来拟合 ${ }^{[97]}$.

$\mathrm{MX}_{2}$ 的拉曼特征也显著地依赖于其层数的变 化 ${ }^{[98 \sim 100]}$. 图3(a)为 1 6层以及体相 $\mathrm{MoS}_{2}$ 的典型拉曼 谱图 ${ }^{[98]}, \mathrm{MoS}_{2}$ 典型的面内振动模 $\mathrm{E}_{2 \mathrm{~g}}{ }^{1}\left(\sim 384 \mathrm{~cm}^{-1}\right)$ 和面 外振动模 $\mathrm{A}_{1 \mathrm{~g}}\left(\sim 405 \mathrm{~cm}^{-1}\right)$ 在所有的谱图均得到了体现, 然而这两个拉曼特征峰随样品层数的变化则有所差 异. 如图3(b)所示, 随着 $\mathrm{MoS}_{2}$ 层数的增加, $\mathrm{E}_{2 \mathrm{~g}}{ }^{1}$ 向低 波数位移, 而 $\mathrm{A}_{1 \mathrm{~g}}$ 向高波数位移, 且 $\mathrm{A}_{1 \mathrm{~g}}$ 峰位随层数的 变化速率约为 $\mathrm{E}_{2 \mathrm{~g}}{ }^{1}$ 模的两倍, 这一反方向位移的现象 是由于层间的长程库伦相互作用力, 同时反映了层 间堆垛引起的结构改变 ${ }^{[98]} . \mathrm{MoS}_{2}$ 的 $\mathrm{A}_{1 \mathrm{~g}}$ 和 $\mathrm{E}_{2 \mathrm{~g}}{ }^{1}$ 振动模 随层数的这一反方向位移的现象使得其频率差成为 $\mathrm{MoS}_{2}$ 层数的判定依据, 1 4层 $\mathrm{MoS}_{2}$ 的 $\mathrm{A}_{1 \mathrm{~g}}$ 和 $\mathrm{E}_{2 \mathrm{~g}}{ }^{1}$ 的频率 
表 1 常见二维材料的带隙、对称性和典型拉曼特征 ${ }^{a)}$

Table 1 Band gap, symmetry and typical Raman modes of selected 2D materials

\begin{tabular}{|c|c|c|c|c|}
\hline 单层材料 & 带隙(eV) & 点群 & 不可约表示 & 典型拉曼特征 \\
\hline 石墨烯 ${ }^{[60]}$ & 0 & $\mathrm{D}_{6 \mathrm{~h}}$ & $\mathrm{~A}_{2 \mathrm{u}}+\mathrm{B}_{2 \mathrm{~g}}+\mathrm{E}_{2 \mathrm{~g}}+\mathrm{E}_{1 \mathrm{u}}$ & $\mathrm{G}\left(\sim 1580 \mathrm{~cm}^{-1}\right), \mathrm{G}^{\prime}\left(\sim 2700 \mathrm{~cm}^{-1}\right)$ \\
\hline 六方氮化嗍 & 4.7 & $\mathrm{D}_{6 \mathrm{~h}}$ & $\mathrm{~A}_{2 \mathrm{u}}+\mathrm{B}_{2 \mathrm{~g}}+\mathrm{E}_{2 \mathrm{~g}}+\mathrm{E}_{1 \mathrm{u}}^{[61]}$ & $\mathrm{E}_{2 \mathrm{~g}}\left(\sim 1370 \mathrm{~cm}^{-1}\right)^{[62]}$ \\
\hline 黑磷 & $1.51^{[63]}$ & $\mathrm{D}_{2 \mathrm{~h}}$ & $2 A_{g}+B_{1 g}+B_{2 g}+2 B_{3 g}+A_{1 u}+2 B_{1 u}+2 B_{2 u}+B_{3 u}^{[56]}$ & $\mathrm{B}_{2 \mathrm{~g}}\left(363 \mathrm{~cm}^{-1}\right), \mathrm{A}_{\mathrm{g}}{ }^{1}\left(440 \mathrm{~cm}^{-1}\right), \mathrm{Ag}_{\mathrm{g}}{ }^{2}\left(471.3 \mathrm{~cm}^{-1}\right)^{[64]}$ \\
\hline $\mathrm{TiS}_{2}(1 \mathrm{~T})$ & 0.02 & $\mathrm{D}_{3 \mathrm{~d}^{3}}$ & $\mathrm{~A}_{1 \mathrm{~g}}+\mathrm{E}_{\mathrm{g}}+2 \mathrm{~A}_{2 \mathrm{u}}+2 \mathrm{E}_{\mathrm{u}}^{[65]}$ & $A_{1 g}\left(232.1 \mathrm{~cm}^{-1}\right), E_{g}\left(333 \mathrm{~cm}^{-1}\right)$ \\
\hline $\mathrm{TiSe}_{2}(1 \mathrm{~T})$ & 0 & $\mathrm{D}_{3 \mathrm{~d}^{3}}$ & $\mathrm{~A}_{1 \mathrm{~g}}+\mathrm{E}_{\mathrm{g}}+2 \mathrm{~A}_{2 \mathrm{u}}+2 \mathrm{E}_{\mathrm{u}}^{[65]}$ & $A_{1 g}\left(205 \mathrm{~cm}^{-1}\right), E_{g}\left(136 \mathrm{~cm}^{-1}\right)^{[66]}$ \\
\hline $\operatorname{TiTe}_{2}(1 \mathrm{~T})$ & 0 & $\mathrm{D}_{3 \mathrm{~d}^{3}}$ & $\mathrm{~A}_{1 \mathrm{~g}}+\mathrm{E}_{\mathrm{g}}+2 \mathrm{~A}_{2 \mathrm{u}}+2 \mathrm{E}_{\mathrm{u}}$ & $\mathrm{A}_{1 \mathrm{~g}}\left(141.5 \mathrm{~cm}^{-1}\right), \mathrm{E}_{\mathrm{g}}\left(124.3 \mathrm{~cm}^{-1}\right)$ \\
\hline $\mathrm{ZrS}_{2}(1 \mathrm{~T})$ & $1.83^{[67]}$ & $D_{3 d^{3}}$ & $\mathrm{~A}_{1 \mathrm{~g}}+\mathrm{E}_{\mathrm{g}}+2 \mathrm{~A}_{2 \mathrm{u}}+2 \mathrm{E}_{\mathrm{u}}^{[67]}$ & $A_{1 \mathrm{~g}}\left(333 \mathrm{~cm}^{-1}\right), E_{\mathrm{g}}\left(251 \mathrm{~cm}^{-1}\right)^{[68]}$ \\
\hline $\mathrm{ZrSe}_{2}(1 \mathrm{~T})$ & 0.4 & $D_{3 d^{3}}$ & $\mathrm{~A}_{1 \mathrm{~g}}+\mathrm{E}_{\mathrm{g}}+2 \mathrm{~A}_{2 \mathrm{u}}+2 \mathrm{E}_{\mathrm{u}}$ & $\mathrm{A}_{1 \mathrm{~g}}\left(194.5 \mathrm{~cm}^{-1}\right), \mathrm{E}_{\mathrm{g}}\left(145.5 \mathrm{~cm}^{-1}\right)^{[69]}$ \\
\hline $\mathrm{ZrTe}_{2}(1 \mathrm{~T})$ & 0 & $\mathrm{D}_{3 \mathrm{~d}}{ }^{3}$ & $\mathrm{~A}_{1 \mathrm{~g}}+\mathrm{E}_{\mathrm{g}}+2 \mathrm{~A}_{2 \mathrm{u}}+2 \mathrm{E}_{\mathrm{u}}$ & $\mathrm{A}_{1 \mathrm{~g}}\left(119.6 \mathrm{~cm}^{-1}\right), \mathrm{E}_{\mathrm{g}}\left(138.5 \mathrm{~cm}^{-1}\right)$ \\
\hline $\mathrm{HfS}_{2}(1 \mathrm{~T})$ & 1.3 & $D_{3 d^{3}}$ & $\mathrm{~A}_{1 \mathrm{~g}}+\mathrm{E}_{\mathrm{g}}+2 \mathrm{~A}_{2 \mathrm{u}}+2 \mathrm{E}_{\mathrm{u}}^{[67]}$ & $A_{1 \mathrm{~g}}\left(337 \mathrm{~cm}^{-1}\right), \mathrm{E}_{\mathrm{g}}\left(262 \mathrm{~cm}^{-1}\right)^{[68]}$ \\
\hline $\mathrm{HfSe}_{2}(1 \mathrm{~T})$ & $1.1^{[70]}$ & $\mathrm{D}_{3 \mathrm{~d}}{ }^{3}$ & $\mathrm{~A}_{1 \mathrm{~g}}+\mathrm{E}_{\mathrm{g}}+2 \mathrm{~A}_{2 \mathrm{u}}+2 \mathrm{E}_{\mathrm{u}}$ & $A_{1 \mathrm{~g}}\left(198 \mathrm{~cm}^{-1}\right), \mathrm{E}_{\mathrm{g}}\left(145 \mathrm{~cm}^{-1}\right)^{[71]}$ \\
\hline $\operatorname{HfTe}_{2}(1 \mathrm{~T})$ & 0 & $\mathrm{D}_{3 \mathrm{~d}}{ }^{3}$ & $\mathrm{~A}_{1 \mathrm{~g}}+\mathrm{E}_{\mathrm{g}}+2 \mathrm{~A}_{2 \mathrm{u}}+2 \mathrm{E}_{\mathrm{u}}$ & $A_{1 g}\left(139.1 \mathrm{~cm}^{-1}\right), E_{g}\left(119.6 \mathrm{~cm}^{-1}\right)$ \\
\hline $\mathrm{VS}_{2}(1 \mathrm{~T})^{[72]}$ & 0 & & & $\mathrm{~A}_{1}\left(330 \mathrm{~cm}^{-1}\right), \mathrm{E}_{2}\left(260 \mathrm{~cm}^{-1}\right)$ \\
\hline $\mathrm{VSe}_{2}(1 \mathrm{~T})$ & $0^{[73]}$ & $D_{3 d^{3}}$ & $\mathrm{~A}_{1 \mathrm{~g}}+\mathrm{E}_{\mathrm{g}}+2 \mathrm{~A}_{2 \mathrm{u}}+2 \mathrm{E}_{\mathrm{u}}$ & $A_{1 g}\left(211 \mathrm{~cm}^{-1}\right), E_{g}\left(143 \mathrm{~cm}^{-1}\right)^{[74]}$ \\
\hline $\mathrm{VTe}_{2}(1 \mathrm{~T})$ & $0^{[75]}$ & $D_{3 d^{3}}$ & $\mathrm{~A}_{1 \mathrm{~g}}+\mathrm{E}_{\mathrm{g}}+2 \mathrm{~A}_{2 \mathrm{u}}+2 \mathrm{E}_{\mathrm{u}}$ & $\mathrm{A}_{1 \mathrm{~g}}\left(121.9 \mathrm{~cm}^{-1}\right), \mathrm{E}_{\mathrm{g}}\left(140.7 \mathrm{~cm}^{-1}\right)$ \\
\hline $\mathrm{NbS}_{2}(2 \mathrm{H})$ & 0 & $\mathrm{D}_{6 \mathrm{~h}}{ }^{4}$ & $\mathrm{~A}_{1 \mathrm{~g}}+2 \mathrm{~A}_{2 \mathrm{u}}+\mathrm{B}_{1 \mathrm{u}}+2 \mathrm{~B}_{2 \mathrm{~g}}+\mathrm{E}_{1 \mathrm{~g}}+2 \mathrm{E}_{1 \mathrm{u}}+\mathrm{E}_{2 \mathrm{u}}+2 \mathrm{E}_{2 \mathrm{~g}}$ & $A_{1 g}\left(379 \mathrm{~cm}^{-1}\right), E_{2 g}{ }^{1}\left(304 \mathrm{~cm}^{-1}\right), E_{1 g}\left(260 \mathrm{~cm}^{-1}\right)^{[76]}$ \\
\hline $\mathrm{NbS}_{2}(3 \mathrm{R})$ & 0 & $\mathrm{C}_{3 \mathrm{v}}{ }^{5}$ & $3 \mathrm{~A}_{1}+3 \mathrm{E}^{[76]}$ & $\begin{array}{l}\mathrm{E}_{1}\left(283 \mathrm{~cm}^{-1}\right), \mathrm{E}_{2}\left(328 \mathrm{~cm}^{-1}\right) \\
\mathrm{A}_{1}\left(387 \mathrm{~cm}^{-1}\right), \mathrm{A}_{2}\left(460 \mathrm{~cm}^{-1}\right)^{[77]}\end{array}$ \\
\hline $\mathrm{NbSe}_{2}(2 \mathrm{H})^{[78]}$ & 0 & $\mathrm{D}_{6 \mathrm{~h}}{ }^{4}$ & $\mathrm{~A}_{1 \mathrm{~g}}+2 \mathrm{~A}_{2 \mathrm{u}}+\mathrm{B}_{1 \mathrm{u}}+2 \mathrm{~B}_{2 \mathrm{~g}}+\mathrm{E}_{1 \mathrm{~g}}+2 \mathrm{E}_{1 \mathrm{u}}+\mathrm{E}_{2 \mathrm{u}}+2 \mathrm{E}_{2 \mathrm{~g}}$ & $\mathrm{~A}_{1 \mathrm{~g}}\left(230.9 \mathrm{~cm}^{-1}\right), \mathrm{E}_{2 \mathrm{~g}}{ }^{1}\left(238.3 \mathrm{~cm}^{-1}\right)$ \\
\hline $\mathrm{NbTe}_{2}\left(1 \mathrm{~T}^{\prime}\right)$ & $0^{[79]}$ & $\mathrm{C}_{2 \mathrm{~h}}{ }^{3}$ & $16 \mathrm{~A}_{\mathrm{g}}+8 \mathrm{~B}_{\mathrm{g}}+9 \mathrm{~A}_{\mathrm{u}}+18 \mathrm{~B}_{\mathrm{u}}^{[80]}$ & \\
\hline $\mathrm{TaS}_{2}(1 \mathrm{~T})$ & 0 & $\mathrm{D}_{3 \mathrm{~d}}$ & $\mathrm{~A}_{1 \mathrm{~g}}+\mathrm{E}_{\mathrm{g}}+2 \mathrm{~A}_{2 \mathrm{u}}+2 \mathrm{E}_{\mathrm{u}}$ & \\
\hline $\mathrm{TaSe}_{2}(1 \mathrm{~T})$ & 0 & $D_{3 d}$ & $\mathrm{~A}_{1 \mathrm{~g}}+\mathrm{E}_{\mathrm{g}}+2 \mathrm{~A}_{2 \mathrm{u}}+2 \mathrm{E}_{\mathrm{u}}$ & $A_{1 g}\left(226 \mathrm{~cm}^{-1}\right), E_{g}\left(159 \mathrm{~cm}^{-1}\right)^{[81]}$ \\
\hline $\mathrm{MoS}_{2}(2 \mathrm{H})$ & $1.86^{[82]}$ & $\mathrm{D}_{3 \mathrm{~h}}$ & $\mathrm{~A}^{\prime}{ }_{1}+\mathrm{E}^{\prime \prime}+2 \mathrm{~A}_{2}^{\prime \prime}+2 \mathrm{E}^{\prime[83]}$ & $\mathrm{A}^{\prime}{ }_{1}\left(404.3 \mathrm{~cm}^{-1}\right), \mathrm{E}^{\prime}\left(384.9 \mathrm{~cm}^{-1}\right)^{[82]}$ \\
\hline $\mathrm{MoSe}_{2}(2 \mathrm{H})$ & $1.57^{[84]}$ & $\mathrm{D}_{3 \mathrm{~h}}$ & $\mathrm{~A}_{1}^{\prime}+\mathrm{E}^{\prime \prime}+2 \mathrm{~A}_{2}{ }_{2}+2 \mathrm{E}^{\prime}$ & $\mathrm{A}^{\prime}{ }_{1}\left(240.6 \mathrm{~cm}^{-1}\right), \mathrm{E}^{\prime}\left(287.1 \mathrm{~cm}^{-1}\right)^{[84]}$ \\
\hline $\operatorname{MoTe}_{2}(2 \mathrm{H})^{[85]}$ & 1.1 & $\mathrm{D}_{6 \mathrm{~h}}{ }^{4}$ & $A_{1 g}+2 A_{2 u}+B_{1 u}+2 B_{2 g}+E_{1 g}+2 E_{1 u}+E_{2 u}+2 E_{2 g}$ & $\mathrm{~A}_{1 \mathrm{~g}}\left(173.3 \mathrm{~cm}^{-1}\right), \mathrm{E}_{2 \mathrm{~g}}{ }^{1}\left(234 \mathrm{~cm}^{-1}\right)$ \\
\hline $\mathrm{WS}_{2}(2 \mathrm{H})$ & $1.99^{[86]}$ & $\mathrm{D}_{3 \mathrm{~h}}{ }^{1}$ & $\mathrm{~A}_{1}^{\prime}+\mathrm{E}^{\prime \prime}+2 \mathrm{~A}_{2}^{\prime \prime}+2 \mathrm{E}^{\prime}$ & $\mathrm{A}^{\prime}{ }_{1}\left(418 \mathrm{~cm}^{-1}\right), \mathrm{E}^{\prime}\left(355 \mathrm{~cm}^{-1}\right)^{[87]}$ \\
\hline $\mathrm{WSe}_{2}(2 \mathrm{H})$ & $1.65^{[84]}$ & $\mathrm{D}_{3 \mathrm{~h}}{ }^{1}$ & $\mathrm{~A}_{1}^{\prime}+\mathrm{E}^{\prime \prime}+2 \mathrm{~A}_{2}^{\prime \prime}+2 \mathrm{E}^{\prime[83]}$ & $\mathrm{A}^{\prime}{ }_{1}\left(249.6 \mathrm{~cm}^{-1}\right), \mathrm{E}^{\prime}\left(249.6 \mathrm{~cm}^{-1}\right)^{[84]}$ \\
\hline $\mathrm{WTe}_{2}\left(\mathrm{~T}_{\mathrm{d}}\right)^{[88]}$ & 0 & $\mathrm{C}_{2 \mathrm{v}}{ }^{7}$ & $11 \mathrm{~A}_{1}+6 \mathrm{~A}_{2}+5 \mathrm{~B}_{1}+11 \mathrm{~B}_{2}$ & \\
\hline $\operatorname{ReS}_{2}\left(1 T^{\prime}\right)^{[89]}$ & 1.62 & $\mathrm{C}_{\mathrm{i}}$ & $18 \mathrm{~A}_{\mathrm{g}}+18 \mathrm{~A}_{\mathrm{u}}$ & \\
\hline $\operatorname{ReSe}_{2}\left(1 \mathrm{~T}^{\prime}\right)^{[89]}$ & 1.31 & $\mathrm{C}_{\mathrm{i}}$ & $18 \mathrm{~A}_{\mathrm{g}}+18 \mathrm{~A}_{\mathrm{u}}$ & \\
\hline $\operatorname{GaSe}(2 \mathrm{H})$ & $3.89^{[90]}$ & $\mathrm{D}_{3 \mathrm{~h}}{ }^{1}$ & $4 \mathrm{~A}_{1}^{\prime}+4 \mathrm{~A}_{2}{ }_{2}+4 \mathrm{E}^{\prime}+4 \mathrm{E}^{\prime \prime 91]}$ & $\begin{array}{l}\mathrm{A}^{\prime}\left(132 \mathrm{~cm}^{-1}\right), \mathrm{E}^{\prime}\left(208 \mathrm{~cm}^{-1}\right) \\
\mathrm{E}^{\prime \prime}\left(230 \mathrm{~cm}^{-1}\right), \mathrm{A}^{\prime}{ }_{1}\left(303.4 \mathrm{~cm}^{-1}\right)^{[28]}\end{array}$ \\
\hline $\mathrm{GaTe}^{[29]}$ & 1.67 & $\mathrm{C}_{2 \mathrm{~h}}{ }^{3}$ & $12 \mathrm{~A}_{\mathrm{g}}+6 \mathrm{~B}_{\mathrm{g}}+6 \mathrm{~A}_{\mathrm{u}}+12 \mathrm{~B}_{\mathrm{u}}$ & \\
\hline $\mathrm{SnS}_{2}(2 \mathrm{H})^{[31]}$ & 2.41 & $\mathrm{D}_{3 \mathrm{~d}}$ & $\mathrm{~A}_{1 \mathrm{~g}}+\mathrm{E}_{\mathrm{g}}+2 \mathrm{~A}_{2 \mathrm{u}}+2 \mathrm{E}_{\mathrm{u}}$ & $\mathrm{A}_{1 \mathrm{~g}}\left(314 \mathrm{~cm}^{-1}\right), \mathrm{E}_{\mathrm{g}}\left(206 \mathrm{~cm}^{-1}\right)$ \\
\hline $\mathrm{SnSe}_{2}(2 \mathrm{H})^{[32]}$ & 1.69 & $\mathrm{D}_{3 \mathrm{~d}}$ & $\mathrm{~A}_{1 \mathrm{~g}}+\mathrm{E}_{\mathrm{g}}+2 \mathrm{~A}_{2 \mathrm{u}}+2 \mathrm{E}_{\mathrm{u}}$ & $\mathrm{A}_{1 \mathrm{~g}}\left(184.1 \mathrm{~cm}^{-1}\right), \mathrm{E}_{\mathrm{g}}\left(108.3 \mathrm{~cm}^{-1}\right)$ \\
\hline $\mathrm{SnSe}$ & $1.3^{[92]}$ & $\mathrm{D}_{2 \mathrm{~h}}{ }^{17}$ & $\begin{array}{l}4 \mathrm{~A}_{\mathrm{g}}+2 \mathrm{~B}_{1 \mathrm{~g}}+4 \mathrm{~B}_{2 \mathrm{~g}}+2 \mathrm{~B}_{3 \mathrm{~g}}+2 \mathrm{~A}_{\mathrm{u}} \\
+4 \mathrm{~B}_{1 \mathrm{u}}+2 \mathrm{~B}_{2 \mathrm{u}}+4 \mathrm{~B}_{3 \mathrm{u}}[3]\end{array}$ & $\begin{array}{l}\mathrm{A}_{\mathrm{g}}{ }^{2}\left(71 \mathrm{~cm}^{-1}\right), \mathrm{B}_{3 \mathrm{~g}^{2}}{ }^{2}\left(108 \mathrm{~cm}^{-1}\right), \\
\mathrm{A}_{\mathrm{g}}{ }^{3}\left(130 \mathrm{~cm}^{-1}\right), \mathrm{Ag}_{\mathrm{g}}{ }^{4}\left(151 \mathrm{~cm}^{-1}\right)^{[94]}\end{array}$ \\
\hline
\end{tabular}

a) 所有未加引用文献的带隙数据摘自文献[20]; 所有未加引用文献的典型拉曼特征摘自文献[75]; 对于拉曼特征峰较多的二维材 料, 此处未详细列出具体的特征峰位, 见表中相应的参考文献

差分别为 $18.7,21.6,23.1$ 和 $24.4 \mathrm{~cm}^{-1}$. 除此之外, $\mathrm{A}_{1 \mathrm{~g}}$ 和 $\mathrm{E}_{2 \mathrm{~g}}{ }^{1}$ 峰的强度也依赖于其层数 ${ }^{[99]}$, 在考虑人射光和 散射光多级反射以及干涉效应的情况下, 这两个特
征峰与 $\mathrm{Si}$ 信号的强度比随着层数增加均表现出单调 增加的趋势, 这一现象同样可以用来定量化 $\mathrm{MoS}_{2}$ 的 层数. 对于其他的 $\mathrm{MX}_{2}$, 如 $\mathrm{MoSe}_{2}, \mathrm{WS}_{2}$ 和 $\mathrm{WSe}_{2}$, 其典 

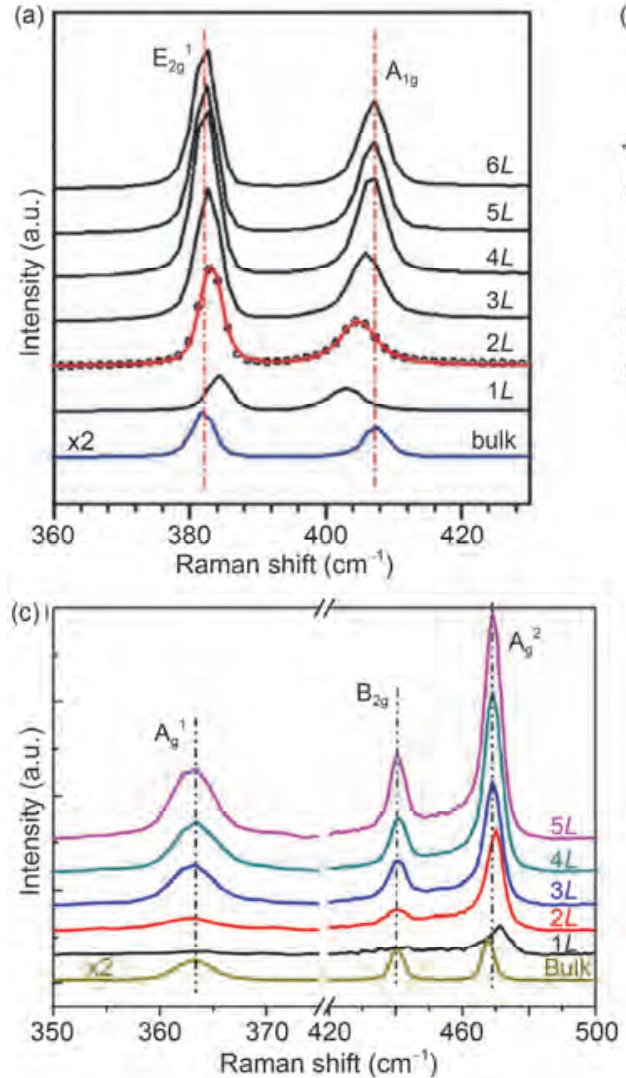
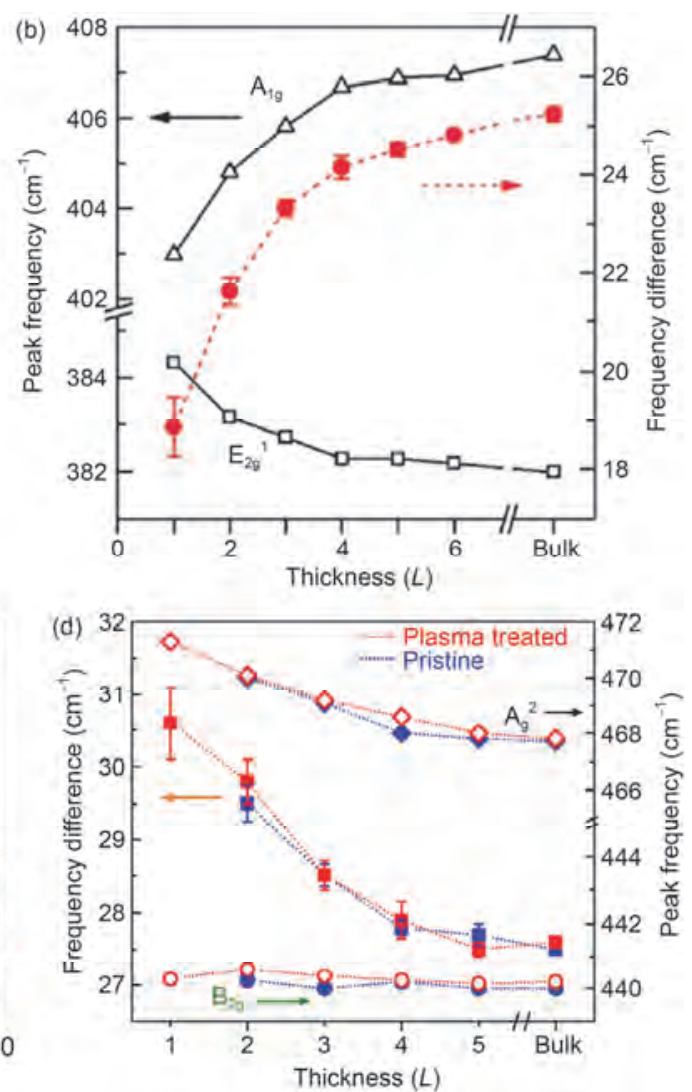

图 3 二维材料拉曼光谱的层数依赖性. (a) 不同厚度 $\mathrm{MoS}_{2}$ 的拉曼谱图 ${ }^{[98]}$; (b) $\mathrm{MoS}_{2}$ 的 $\mathrm{A}_{1 \mathrm{~g}}$ 和 $\mathrm{E}_{2 \mathrm{~g}}{ }^{1}$ 振动模峰位及其峰位差的层数依赖性 ${ }^{[98]}$; (c) 不 同层数黑磷的拉曼谱图 ${ }^{[64]}$; (d) 黑磷的 $\mathrm{A}_{\mathrm{g}}{ }^{2}$ 和 $\mathrm{B}_{2 \mathrm{~g}}$ 拉曼峰位及其频率差随层数的变化关系 ${ }^{[64]}$

Figure 3 Thickness dependence of Raman spectroscopy of 2D materials. (a) Raman spectra of $\mathrm{MoS}_{2}$ with different thickness ${ }^{[98]}$. (b) Thickness-dependent peak position and frequency of $\mathrm{A}_{1 \mathrm{~g}}$ and $\mathrm{E}_{2 \mathrm{~g}}{ }^{1}$ modes of $\mathrm{MoS}_{2}{ }^{[98]}$. (c) Raman spectra of black phosphorus with different thickness ${ }^{[64]}$. (d) Peak position and frequency difference of $\mathrm{A}_{\mathrm{g}}{ }^{2}$ and $\mathrm{B}_{2 \mathrm{~g}}$ modes as a function of the number of layers in black phosphorus ${ }^{[64]}$

型拉曼特征峰 $\mathrm{A}_{1 \mathrm{~g}}$ 和 $\mathrm{E}_{2 \mathrm{~g}}{ }^{1}$ 的频率均随着材料层数增加 向反方向位移, 同样可以通过拉曼光谱对其层数进 行快速准确的判定.

同样，黑磷的拉曼特征峰也有一定的层数依赖 性 ${ }^{[64,101]}$. 图3(c)为不同厚度的黑磷的拉曼谱图, $\mathrm{A}_{\mathrm{g}}{ }^{2}$ 和 $\mathrm{B}_{2 \mathrm{~g}}$ 振动模的峰位及频率差随层数的变化关系示于图 3(d)中, 从图中可以看出, 随着黑磷层数的增加, 面 内振动模 $\mathrm{A}_{\mathrm{g}}{ }^{2}$ 明显地向低波数位移, 而 $\mathrm{A}_{\mathrm{g}}{ }^{1}$ 和 $\mathrm{B}_{2 \mathrm{~g}}$ 的峰 位则几乎没有位移 ${ }^{[64]}$. 根据耦合谐振子模型, 随着 二维材料层数的增加, 其拉曼特征峰会由于附加的 层间范德华相互作用而向高波数位移. 黑磷是一种 各向异性的层状材料, 不同方向的晶格参数对外界 的响应会有所不同, 研究表明黑磷扶手椅型 (armchair)方向的晶格参数随着层数的变化显著地发 生改变, 而其他方向的晶格参数则几乎保持不变, 这 一各向异性的结构特点导致了黑磷振动模的反常位
移行为.

\section{3 拉曼光谱法测定二维材料的边缘手性/晶 格取向}

\section{1 拉曼光谱法测定二维材料的边缘手性}

根据石墨烯的晶格结构, 其手性的边缘分为锯 齿型(zigzag)和扶手椅型(armchair)两种基本类型. 研 究表明 ${ }^{[47,102 ~ 104]}$, 石墨烯的拉曼 D峰与其边缘手性密 切相关, 在armchair边缘处, 其缺陷波矢可以连接布 里渊区的两个不等价的狄拉克点 $\mathrm{K}$ 和 $\mathrm{K}^{\prime}$, 使得拉曼散 射过程中的动量守恒可以得到满足, 从而 $\mathrm{D}$ 峰的双共 振散射过程可以发生. 而在zigzag边缘处动量不守恒 导致 $\mathrm{D}$ 峰被禁阻, 因此石墨烯的 armchair边缘对 $\mathrm{D}$ 峰 的贡献较zigzag边缘大. 除此之外, 当人射光为线偏 振光时, 石墨烯边缘处拉曼 $\mathrm{D}$ 峰的产生还与人射光的 
偏振方向有关, 只有当人射激光的偏振方向沿着石 墨烯的边缘方向时, armchair边缘处才可以产生较强 的D峰信号, 据此可以利用偏振拉曼光谱对石墨烯的 边缘手性进行判定.

在二维材料的边缘处, 由于对称性的破缺, 其原 子结构也会发生重构, 导致其拉曼选择定则会发生 变化. 对于二维黑磷, 根据其拉曼张量的形式以及拉 曼散射强度的计算可知, 在激光垂直人射的情况 (激 光沿着 $Y$ 方向传播)下, 当人射激光和散射光的偏振 方向 $e_{\mathrm{i}}$ 和 $e_{\mathrm{s}}$ 平行且均沿着其 $\operatorname{armchair}(Z)$ 或者 $\operatorname{zigzag}(X)$ 晶格方向 (即 $Z Z$ 或者 $X X$ 散射模式)时, $A_{\mathrm{g}}$ 模可以检测 到, 当 $e_{\mathrm{i}}$ 和 $e_{\mathrm{s}}$ 垂直且沿着其晶格方向 (即 $X Z$ 或者 $Z X$ 散 射模式, 这两者是一致的)时, $\mathrm{B}_{2 \mathrm{~g}}$ 模可以被检测到, 而在这一散射模式下, $B_{1 g}$ 和 $B_{3 g}$ 模是被禁阻的 ${ }^{[105]}$ (如 图4(b)中上面两条谱线). 然而, 在二维黑磷的边缘 处, 这一常规的拉曼选择定则不再适用. 从图4(b)中 粉色谱线和4(c)中可以看出, 全对称振动模 $\mathrm{A}_{\mathrm{g}}{ }^{1}$ 和 $\mathrm{A}_{\mathrm{g}}{ }^{2}$ 在交叉偏振 $X Z$ 下可以出现, 且 $\mathrm{A}_{\mathrm{g}}{ }^{1}$ 在zigzag边缘处较 强, $\mathrm{A}_{\mathrm{g}}{ }^{2}$ 在armchair边缘处较强; $\mathrm{B}_{2 \mathrm{~g}}$ 振动模出现在平行 偏振ZZ下的zigzag边缘处和 $X X$ 下的 armchair边缘处; 原本被禁阻的 $B_{1 \mathrm{~g}}$ 和 $\mathrm{B}_{3 \mathrm{~g}}{ }^{1}$ 模在平行偏振和交叉偏振下 均可以出现, $\mathrm{B}_{1 \mathrm{~g}}$ 在 $X Z$ 下的 zigzag 边缘和 $X X$ 下的 armchair边缘处较强, 而 $\mathrm{B}_{3 \mathrm{~g}}{ }^{1}$ 在 $X Z$ 下的 armchair边缘 和ZZ下的zigzag边缘处较强 ${ }^{[105]}$. 黑磷边缘处的这一 反常拉曼是由于其边缘处的原子重构引起的边缘声 子态的出现, 导致其振动模拉曼张量原来的一些零 矩阵元变为非零矩阵元, 从而其拉曼选择定则发生 变化. 据此, 可以通过偏振拉曼光谱对二维黑磷的边 缘手性进行判定. 对于其他的二维材料, 目前没有系 统的关于其边缘拉曼光谱的研究, 在此不做赘述.

\section{2 拉曼光谱法测定各向同性二维材料的晶格 方向}

众所周知，对于石墨烯、MoS 2 等面内各向同性的 二维材料, 其拉曼特征峰的强度不随着人射光偏振 方向和晶格方向夹角的变化而改变. 而当给二维材 料施加单轴应力时, 其拉曼特征峰会发生位移, 当应 力大小达到一定程度时, 其双重简并的拉曼特征峰 发生去简并从而发生裂分, 且裂分的两个峰具有不 同的偏振依赖性，据此可以利用偏振拉曼对各向同 性的二维材料的晶格方向进行指认 ${ }^{[106 ~ 108]}$. 这里首先 对偏振拉曼中的一些方向进行定义(图 5(a)), $x$ 和 $y$ 分 别为石墨烯/ $\mathrm{MoS}_{2}$ 的zigzag和armchair方向, 红色箭头 为应力的施加方向, 紫色和蓝色箭头分别代表人射
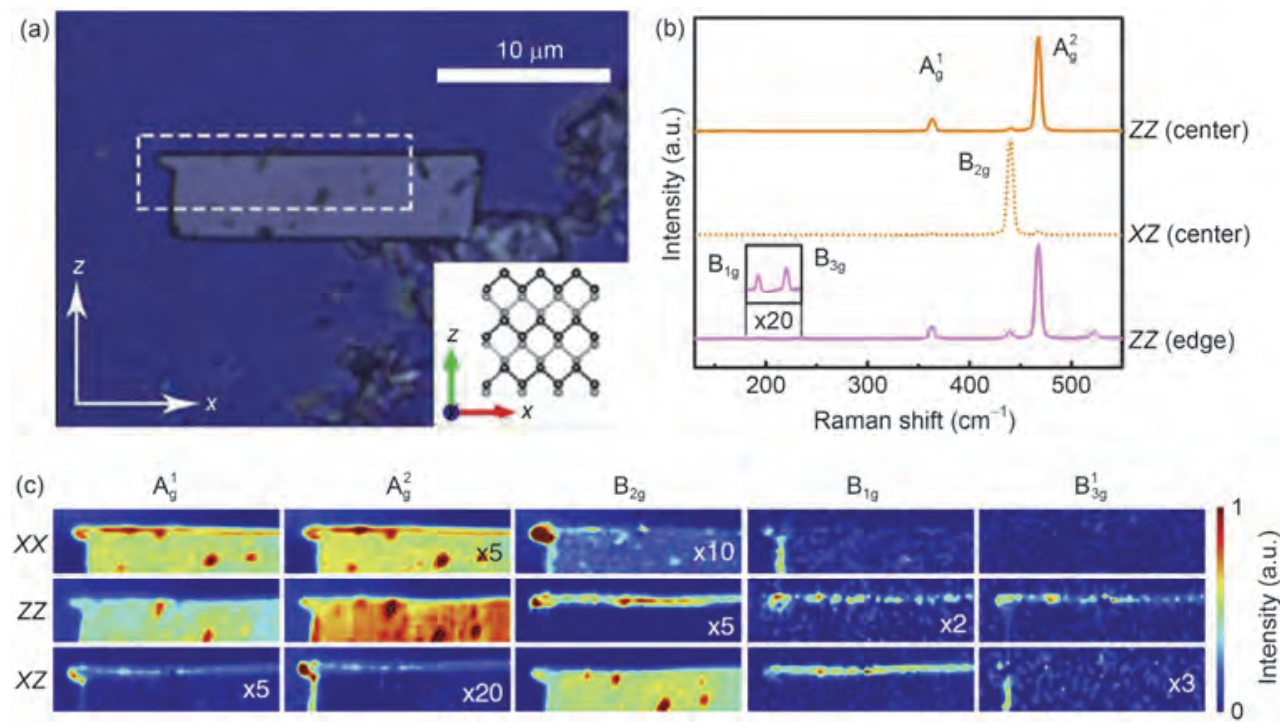

图 4 二维黑磷边缘的异常偏振拉曼光谱. (a) 二维黑磷样品的光学照片, 样品的垂直方向 $Z$ 和水平方向 $X$ 分别为其armchair和zigzag晶格方向; (b) $Z Z$ 和 $X Z$ 散射模式下样品中心的拉曼谱图和 $Z Z$ 模式下 armchair边缘处的拉曼谱图; (c) $X X, Z Z$ 和 $X Z$ 散射模式下黑磷的 $\mathrm{A}_{\mathrm{g}}{ }^{1}, \mathrm{~A}_{\mathrm{g}}{ }^{2}, \mathrm{~B}_{2 \mathrm{~g}}, \mathrm{~B}_{1 \mathrm{~g}}$ 和 $\mathrm{B}_{3 \mathrm{~g}}{ }^{1}$ 模 的强度成像 ${ }^{[105]}$

Figure 4 Anomalous polarized Raman spectroscopy in 2D black phosphorous edges. (a) Optical image of a black phosphorus flake. Perpendicular direction $Z$ and horizontal direction $X$ represent the armchair and zigzag crystalline orientations, perspectively. (b) Polarized Raman spectra at the center under $Z Z$ and $X Z$ scattering configurations and at armchair edge with $Z Z$ scattering configuration. (c) Raman intensity images of $A_{g}{ }^{1}, A_{g}{ }^{2}, B_{2 g}, B_{1 g}$ and $\mathrm{B}_{3 \mathrm{~g}}{ }^{1}$ modes with $X X, Z Z$ and $X Z$ scattering configurations ${ }^{[105]}$ 

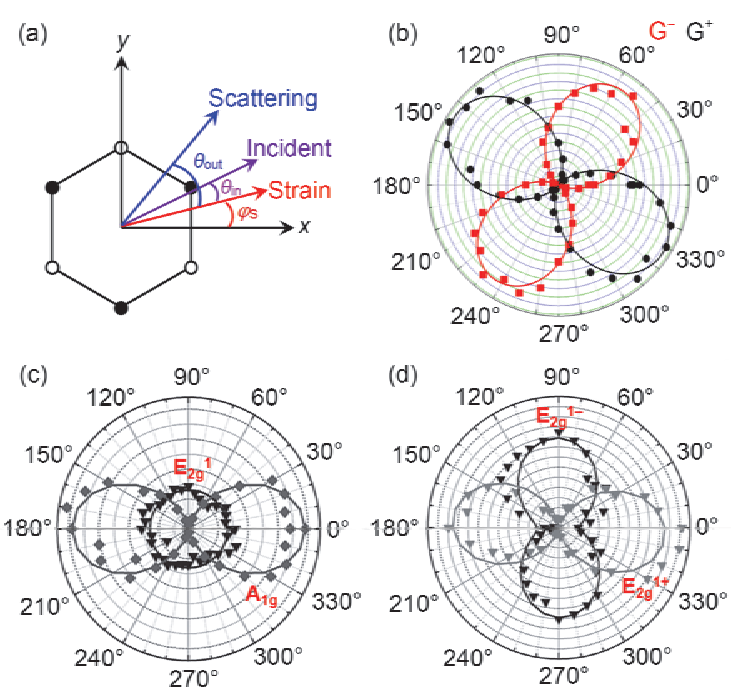

图 5 应力作用下各向同性二维材料拉曼强度的偏振依赖性. (a) 应 力下偏振拉曼光学配置示意图 ${ }^{[106]}$; (b) 散射光偏振方向沿着应力施 加方向 $\left(\theta_{\text {out }}=0^{\circ}\right)$ 时, 石墨烯的 $\mathrm{G}^{+}$和 $\mathrm{G}^{-}$峰强度随着人射光偏振方向与应 力施加方向之间夹角 $\theta_{\mathrm{in}}$ 的变化关系 ${ }^{[106]}$; (c) 未施加应力时, $\mathrm{MoS}_{2}$ 的 $\mathrm{A}_{1 \mathrm{~g}}$ 和 $\mathrm{E}_{2 \mathrm{~g}}{ }^{1}$ 模的强度随人射光和散射光偏振方向之间的夹角的变化关 系 ${ }^{[108]}$; (d) 在 3.6\%的单轴应力作用下, $\theta_{\mathrm{in}}=0^{\circ}$ 时 $\mathrm{MoS}_{2}$ 的 $\mathrm{E}_{2 \mathrm{~g}}{ }^{1+}$ 和 $\mathrm{E}_{2 \mathrm{~g}}{ }^{1-}$ 峰 强度随 $\theta_{\text {out }}$ 的变化关系 ${ }^{[108]}$

Figure 5 Polarization dependence of Raman intensity of isotropic 2D materials under strain. (a) Polarization geometry under $\operatorname{strain}^{[106]}$. (b) Polar plot of $\mathrm{G}^{+}$and $\mathrm{G}^{-}$intensity of graphene as a function of the angle between the polarization direction of incident light and strain axis $\theta_{\mathrm{in}}$ when the scattered light polarization is along with the strain axis $\left(\theta_{\text {out }}=0^{\circ}\right)^{[106]}$. (c) Intensity of $\mathrm{A}_{1 \mathrm{~g}}$ and $\mathrm{E}_{2 \mathrm{~g}}{ }^{1}$ modes in $\mathrm{MoS}_{2}$ as a function of the angle between the polarization direction of incident and scattered light ${ }^{[108]}$. (d) Polar plot of $\mathrm{E}_{2 \mathrm{~g}}{ }^{1+}$ and $\mathrm{E}_{2 \mathrm{~g}}{ }^{1-}$ intensity as a function of $\theta_{\text {out }}$ with $\theta_{\mathrm{in}}=0^{\circ}$ under the uniaxial strain of $3.6 \%^{[108]}$

光 $e_{\mathrm{i}}$ 和散射光 $e_{\mathrm{s}}$ 的偏振方向, $\varphi_{\mathrm{s}}$ 为应力施加方向与 zigzag方向之间的夹角, $\theta_{\text {in }}$ 和 $\theta_{\text {out }}$ 分别为应力施加方向 与 $e_{\mathrm{i}}$ 和 $e_{\mathrm{s}}$ 之间的夹角 ${ }^{[106]}$. 在应力作用下, 石墨烯的 $\mathrm{G}^{+}$ 和 $\mathrm{G}^{-}$峰强度与 $\theta_{\mathrm{in}}, \theta_{\mathrm{out}}$ 和 $\varphi_{\mathrm{s}}$ 之间有如下的函数关系:

$$
I_{\mathrm{G}^{-}} \propto \sin ^{2}\left(\theta_{\text {in }}+\theta_{\text {out }}+3 \varphi_{\mathrm{s}}\right), I_{\mathrm{G}^{+}} \propto \cos ^{2}\left(\theta_{\text {in }}+\theta_{\text {out }}+3 \varphi_{\mathrm{s}}\right) .
$$

根据以上关系式, 对 $\mathrm{G}^{+}$和 $\mathrm{G}^{-}$峰强度进行拟合, 即可得到 $\varphi_{\mathrm{s}}$ 值, 从而利用应力下的偏振拉曼光谱对 石墨烯的晶格方向进行指认. 如图 5(b) 所示, 在 $\theta_{\mathrm{out}}=0^{\circ}$ 时, 随着 $\theta_{\mathrm{in}}$ 的变化, $\mathrm{G}^{+}$和 $\mathrm{G}^{-}$峰强度均表现出 $180^{\circ}$ 的周期性变化, 且两者的变化规律正好相反, 对 此进行拟合, 可得 $\varphi_{\mathrm{s}}=11.3^{\circ[106]}$. 石墨烯的 $\mathrm{G}^{\prime}$ 峰在应力 作用下也会发生裂分, 且 $\mathrm{G}^{\prime}$ 和 $\mathrm{G}^{\prime}$ 峰的强度也依赖于 $\theta_{\text {out }}, \theta_{\text {in }}$ 和 $\varphi_{\mathrm{s}}^{[107]}$, 据此也可以对石墨烯的晶格方向进 行指认.

$\mathrm{MX}_{2}(\mathrm{M}=\mathrm{Mo}, \mathrm{W} ; \mathrm{X}=\mathrm{S}, \mathrm{Se})$ 也是一类面内各向同
性的二维材料, 根据拉曼选择定则, $\mathrm{A}_{1 \mathrm{~g}}$ 振动模仅可 以在平行偏振下检测到, 而 $\mathrm{E}_{2 \mathrm{~g}}{ }^{1}$ 在平行和交叉偏振下 均可以检测到, 因此在人射光和散射光的偏振方向 之间的夹角发生变化时, $\mathrm{A}_{1 \mathrm{~g}}$ 强度表现出 $180^{\circ}$ 的周期 性变化, 而 $\mathrm{E}_{2 \mathrm{~g}}{ }^{1}$ 则保持强度不变 ${ }^{[108]}$ (图 5(c)). 在应力 作用下, $\mathrm{A}_{1 \mathrm{~g}}$ 仍表现为与未施加应力时类似的偏振依 赖性, 而 $E_{2 \mathrm{~g}}{ }^{1}$ 不再简并而发生裂分, 且 $E_{2 \mathrm{~g}}{ }^{1+}$ 和 $E_{2 \mathrm{~g}}{ }^{1-}$ 强 度与 $\theta_{\mathrm{out}}, \theta_{\mathrm{in}}$ 和 $\varphi_{\mathrm{s}}$ 之间的变化关系与石墨烯的 $\mathrm{G}$ 峰类 似, 据此同样可以指认 $\mathrm{MoS}_{2}$ 的晶格方向 ${ }^{[108]}$. 图 5(d) 所示样品的应力施加方向正好与其zigzag晶格方向 非常接近.

\section{3 拉曼光谱法测定各向异性二维材料的晶格 方向}

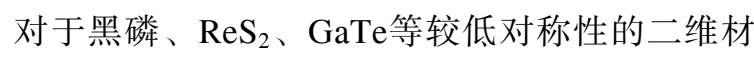
料, 结构上的各向异性决定了其光学、电学、机械等 性质上的各向异性，拉曼光谱也不例外.

这里仅考虑二维材料面内的各向异性, 而不考 虑在垂直于二维材料平面的方向上的各向异性. 当 $e_{\mathrm{i}}$ 和 $e_{\mathrm{s}}$ 与二维材料的晶格方向之间的夹角分别为 $\theta$ 和 $\theta^{\prime}$, 则 $e_{\mathrm{i}}$ 和 $e_{\mathrm{s}}$ 可以表示为如下形式:

$$
e_{\mathrm{i}}=\left(\begin{array}{lll}
\cos \theta & \sin \theta & 0
\end{array}\right), e_{\mathrm{s}}=\left(\begin{array}{lll}
\cos \theta^{\prime} & \sin \theta^{\prime} & 0
\end{array}\right)^{\mathrm{T}} .
$$

在平行偏振情况下 $\theta^{\prime}=\theta$, 在交叉偏振情况下 $\theta^{\prime}=\theta+90^{\circ}$. 然后再通过式(1)计算振动模的拉曼散射 强度. 通过对不同晶系的振动模的拉曼散射强度计 算发现，属于三斜、单斜以及正交晶系的材料均具有 各向异性的拉曼光谱.

黑磷是一种类石墨的层状材料具有褶皱的蜂窝 状结构 ${ }^{[109]}$ (图6(a)), 属于正交晶系, 具有 $\mathrm{D}_{2 \mathrm{~h}}$ 点群对 称性 ${ }^{[56,110]}$. 背散射模式下, 只有 $\mathrm{A}_{\mathrm{g}}$ 和 $\mathrm{B}_{2 \mathrm{~g}}$ 模可以被检 测到, 定义 $e_{\mathrm{i}}$ 和黑磷的 armchair方向之间夹角为 $\theta$, 则 平行偏振和交叉偏振下黑磷的 $\mathrm{A}_{\mathrm{g}}$ 和 $\mathrm{B}_{2 \mathrm{~g}}$ 模的拉曼信号 强度具有如下形式:

$$
\begin{array}{ll}
S_{\|}^{\mathrm{A}_{\mathrm{g}}} \propto\left|a \sin ^{2} \theta+c \cos ^{2} \theta\right|^{2}, & S_{\perp}^{\mathrm{A}_{\mathrm{g}}} \propto|(a-c) \sin \theta \cos \theta|^{2}, \\
S_{\|}^{\mathrm{B}_{28}} \propto|e \sin 2 \theta|^{2}, & S_{\perp}^{\mathrm{B}_{2 g}} \propto|e \cos 2 \theta|^{2} .
\end{array}
$$

根据以上关系, 即可在平行偏振下根据 $\mathrm{A}_{\mathrm{g}}$ 模的 周期性变化对黑磷的晶格方向进行指认. 研究表明, 在平行偏振下, $\mathrm{A}_{\mathrm{g}}{ }^{2}$ 模强度在 $e_{\mathrm{i}}$ 平行于黑磷样品的 armchair方向时为较大的极大值, 而在 $e_{\mathrm{i}}$ 平行于黑磷 样品的zigzag方向时为较小的极大值 ${ }^{[109]}$ (图6(b)). 

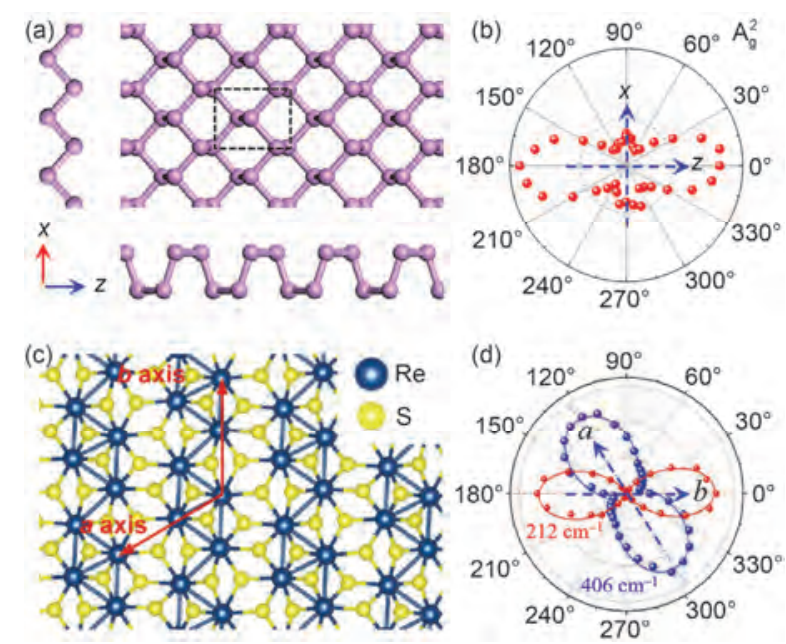

图 6 各向异性二维材料偏振拉曼强度的晶格方向依赖性. (a) 黑磷 的晶体结构示意图 ${ }^{[109]}$; (b) 平行偏振下, 黑磷的 $\mathrm{A}_{\mathrm{g}}{ }^{2}$ 模强度随 $e_{\mathrm{i}}$ 与其 armchair方向之间夹角的变化关系 ${ }^{[109]}$; (c) $\operatorname{ReS}_{2}$ 的晶体结构示意图 ${ }^{[89]}$; (d) 平行偏振下, $\mathrm{ReS}_{2}$ 的 212 和 $406 \mathrm{~cm}^{-1}$ 模强度随 $e_{\mathrm{i}}$ 与其 $b$ 轴方向之间 夹角的变化关系 ${ }^{[89]}$

Figure 6 Crystalline orientation dependence of Raman intensity of anisotropic 2D materials. (a) Crystal structure of black phosphorus ${ }^{[109]}$. (b) Polar plot of $\mathrm{A}_{\mathrm{g}}{ }^{2}$ intensity as a function of the angle between $e_{\mathrm{i}}$ and armchair direction of BP with parallel polarization configuration ${ }^{[109]}$. (c) Crystal structure of $\mathrm{ReS}_{2}{ }^{[89]}$. (d) Polar plot of 212 and $406 \mathrm{~cm}^{-1}$ intensity as a function of the angle between $e_{\mathrm{i}}$ and $b$ axis of $\mathrm{ReS}_{2}$ with parallel polarization configuration ${ }^{[89]}$

$\mathrm{ReS}_{2}$ 的稳定结构为扭曲的八面体相, 形成了变 形的 S原子层和zigzag的 $\operatorname{Re}$ 原子链 $(b \text { 轴 })^{[89]}$, 其结构示 意图如图 6(c). $\operatorname{ReS}_{2}$ 属于三斜晶系 $\mathrm{C}_{\mathrm{i}}$, 所有的拉曼活 性的振动模均为全对称振动模 $\mathrm{A} g$, 定义 $\theta$ 为 $e_{\mathrm{i}}$ 和 $\mathrm{ReS}_{2}$ 的 Re原子链 ( $b$ 轴) 方向之间的夹角, 则平行和交叉偏 振下 $A_{g}$ 的强度可以表示为

$$
\begin{aligned}
& S_{\|}^{\mathrm{Ag}_{\mathrm{g}}} \propto\left|a \cos ^{2} \theta+b \sin ^{2} \theta+d \sin 2 \theta\right|^{2}, \\
& S_{\perp}^{\mathrm{A}_{\mathrm{g}}} \propto|(b-a) \sin \theta \cos \theta+d \cos 2 \theta|^{2} .
\end{aligned}
$$

研究表明, 平行偏振下, 当位于 $212 \mathrm{~cm}^{-1}$ 的拉曼 峰最强时, $\operatorname{ReS}_{2}$ 的 $\operatorname{Re}$ 原子链 ( $b$ 轴)正好与 $e_{\mathrm{i}}$ 平行, 而当 位于 $406 \mathrm{~cm}^{-1}$ 的拉曼峰最强时, $\operatorname{ReS}_{2}$ 的 $a$ 轴与 $e_{\mathrm{i}}$ 平 行 $^{[89]}$ (图6(d)).

同样地, 正交晶系的 $\mathrm{SnSe}^{[111]}$ 和 $\mathrm{T}_{\mathrm{d}}-\mathrm{WTe}_{2}{ }^{[88]}$ 、单斜 晶系的 $\mathrm{GaTe}^{[112]}$ 和 $1 \mathrm{~T}^{\prime}-\mathrm{MoTe}_{2}{ }^{[113]}$ 、三斜晶系的 $\mathrm{ReSe}_{2}{ }^{[114]}$ 和 $\mathrm{ReS}_{2(1-x)} \mathrm{Se}_{2 x}{ }^{[89]}$ 也具有各向异性的拉曼光 谱, 偏振拉曼光谱同样可以对其晶格方向进行指认.

然而, 仍有一些问题通过简单的拉曼张量的分 析无法解释, 比如: 黑磷的 $\mathrm{A}_{\mathrm{g}}{ }^{2}$ 模强度在平行偏振下 会出现两个大小不一的极大值, 而根据其拉曼散射
强度的计算公式无法对其进行拟合; $\mathrm{ReS}_{2}$ 的偏振拉曼 通常会出现异常. 这可能是由于这些材料的各向异 性的吸收 ${ }^{[33,115]}$ 以及人射光和散射光在各向异性材料 中的双折射效应 ${ }^{[16]}$ 导致的. 目前已经有一些工作在 考虑各向异性吸收与双折射效应的情况下对黑磷 ${ }^{[117]}$ 和 $\mathrm{ReS}_{2}{ }^{[118]}$ 的拉曼选择定则进行了修正, 研究表明激 光波长、样品厚度以及振动模的类型均会对各向异性 二维材料的拉曼选择定则产生影响, 在此就不做详 细介绍了.

\section{4 拉曼光谱法测定二维合金材料的成分}

$\mathrm{MX}_{2}(\mathrm{M}=\mathrm{Mo}, \mathrm{W} ; \mathrm{X}=\mathrm{S}, \mathrm{Se})$ 具有类似的原子结构, 其带隙随着金属元素或者硫族元素的不同而有所不 同, 将两种不同带隙的 $\mathrm{MX}_{2}$ 合金化，即可通过组分的 变化连续调控二维合金材料的导带和价带位置, 从而 对其带隙进行可控调控 ${ }^{[119]}$. 当然, 其拉曼特征峰的位 置也会随着合金组分变化而变化 ${ }^{[84,87,120]}$ (图7). 在单层 合金材料中, 随着材料组分的变化, 不同的拉曼振动 模会表现出不同的行为, 通常情况下, 金属元素变化 的二维合金材料(如 $\mathrm{Mo}_{1-x} \mathrm{~W}_{x} \mathrm{~S}_{2}{ }^{[86,87,121]}, \mathrm{Mo}_{1-x} \mathrm{~W}_{x} \mathrm{Se}_{2}{ }^{[84]}$ ) 的全对称振动模 $\mathrm{A}_{1 \mathrm{~g}}$ (在单层中表现为 $\mathrm{A}^{\prime}{ }_{1}$ ) 会表现出单 模行为, 双重简并的振动模 $\mathrm{E}_{2 \mathrm{~g}}{ }^{1}$ (在单层中表现为 $\mathrm{E}^{\prime}$ ) 会表现出双模行为, 二阶振动模均表现出双模行 为 ${ }^{[84,87]}$ (图 7(a)和 (c)); 而硫族元素变化的二维合金材 料(如 $\mathrm{MoS}_{2(1-x)} \mathrm{Se}_{2 x}{ }^{[82,122]}, \mathrm{ReS}_{2(1-x)} \mathrm{Se}_{2 x}{ }^{[89]}$ ) 的拉曼振动 模则一般表现出双模行为 ${ }^{[120]}$ (图7(b)). 这里, 单模行 为是指两个端材料的这一振动模在所有组分的样品 中均表现为一个峰, 双模行为则是两个端材料的这 一振动模在合金样品中表现为两个峰.

改进的随机元素等位移模型(MREI)可以用来预 测和拟合合金材料的晶格振动模，这一模型是基于 以下两个假设：(1) 合金中相同类型的原子以相同的 振幅和相位振动; (2) 合金元素在合金中是随机分布 的. 对于单层 $\mathrm{Mo}_{1-x} \mathrm{~W}_{x} \mathrm{~S}_{2}{ }^{[87]}, \mathrm{A}_{1 \mathrm{~g}}$ 是两个 $\mathrm{S}$ 原子在面外 的反向振动产生的面外振动模, 基于 MREI模型, $\mathrm{Mo}_{1-x} \mathrm{~W}_{x} \mathrm{~S}_{2}$ 的 $\mathrm{A}_{1 \mathrm{~g}}$ 模的原子位移方程可以表示为

$$
m_{\mathrm{s}} \ddot{\mu}_{\mathrm{s}}=-(1-x) F_{1} \mu_{\mathrm{s}}-x F_{2} \mu_{\mathrm{s}},
$$

式中 $m_{\mathrm{S}}$ 和 $\mu_{\mathrm{S}}$ 分别为 $\mathrm{S}$ 原子的质量和位移, $F_{1}$ 和 $F_{2}$ 分别 为 Mo-S 和W-S 在坚直方向相互作用的力常数. 在力 常数与 $x$ 成线性关系以及长波极限的假设下, $\mathrm{A}_{1 \mathrm{~g}}$ 的频 率可以表示为 

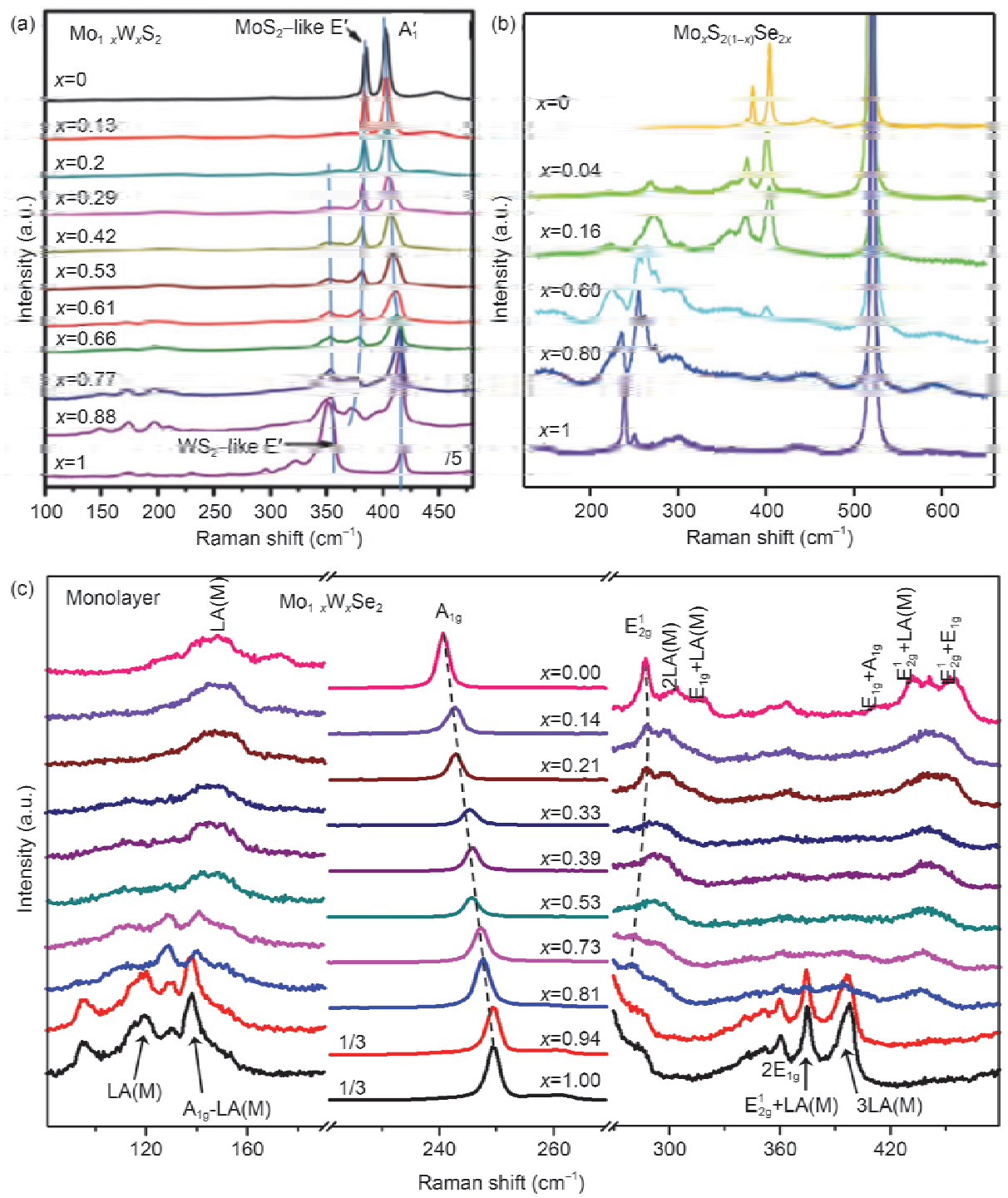

图 7 单层 $\mathrm{Mo}_{1-x} \mathrm{~W}_{x} \mathrm{~S}_{2}{ }^{[87]}(\mathrm{a}), \mathrm{MoS}_{2(1-x)} \mathrm{Se}_{2 x}{ }^{[120]}(\mathrm{b})$ 和 $\mathrm{Mo}_{1-x} \mathrm{~W}_{x} \mathrm{Se}_{2}{ }^{[84]}(\mathrm{c})$ 的组分依赖的拉曼谱图

Figure 7 Composition-dependent Raman spectra of monolayer $\mathrm{Mo}_{1-x} \mathrm{~W}_{x} \mathrm{~S}_{2}{ }^{[87]}$ (a), $\operatorname{MoS}_{2(1-x)} \mathrm{Se}_{2 x}{ }^{[120]}$ (b) and $\mathrm{Mo}_{1-x} \mathrm{~W}_{x} \mathrm{Se}_{2}{ }^{[84]}$ (c)

$$
\begin{aligned}
\omega_{\mathrm{A}_{1 g}} & =\left[\frac{(1-x) F_{1}+x F_{2}}{m_{\mathrm{S}}}\right]^{1 / 2} \\
& =\left[\frac{(1-x)(1-\theta x) F_{10}+x(1-\theta x) F_{20}}{m_{\mathrm{S}}}\right]^{1 / 2},
\end{aligned}
$$

其中 $F_{10}$ 和 $F_{20}$ 分别为 $F_{1}$ 和 $F_{2}$ 在 $x$ 趋于 0 时的力常数, $\theta$ 为 与合金中力常数相关的一个参量. 根据 $x=0$ 和 $x=1$ 时 的实验值, 利用最小二乘法对式(7)进行拟合, 即可 得到组分依赖的 $\mathrm{A}_{1 \mathrm{~g}}$ 的拉曼峰位, 同时可以得到力常 数 $F_{1}$ 和 $F_{2}$. 这一方法同样适用于双重简并的 $\mathrm{E}_{2 \mathrm{~g}}{ }^{1}$ 模以 及 $\mathrm{Mo}_{1-x} \mathrm{~W}_{x} \mathrm{Se}_{2}$ 合金的拉曼振动模. 单层 $\mathrm{Mo}_{1-x} \mathrm{~W}_{x} \mathrm{~S}_{2}$ 和 $\mathrm{Mo}_{1-x} \mathrm{~W}_{x} \mathrm{Se}_{2}$ 合金的 $\mathrm{A}_{1 \mathrm{~g}}$ 模与其组分具有如下的关系:

$$
\begin{aligned}
& \mathrm{Mo}_{1-x} \mathrm{~W}_{x} \mathrm{~S}_{2}: \omega_{\mathrm{A}_{1 \mathrm{~g}}}=401.6\left(1+0.080 x+0.002 x^{2}\right)^{1 / 2}, \\
& \mathrm{Mo}_{1-x} \mathrm{~W}_{x} \mathrm{Se}_{2}: \omega_{\mathrm{A}_{1 \mathrm{~g}}}=240.1\left(1+0.115 x-0.040 x^{2}\right)^{1 / 2} .
\end{aligned}
$$

据此，可以通过拉曼光谱对未知含量的合金的 组分进行定量. 表2列出了二维单层合金材料的能带 参数和组分依赖的拉曼峰位.

\section{5 拉曼光谱法测定二维材料的缺陷密度和 掺杂浓度}

对于含有缺陷的石墨烯，通常会出现位于 1350 $\mathrm{cm}^{-1}$ 的拉曼 $\mathrm{D}$ 峰和 $1620 \mathrm{~cm}^{-1}$ 的 $\mathrm{D}^{\prime}$ 峰, 这两个缺陷峰分 别产生于谷间和谷内散射过程, 其中 $\mathrm{D}$ 峰和 $\mathrm{G}$ 峰的强 


\section{表 2 二维单层合金材料的能带参数和组分依赖的拉曼峰位}

Table 2 Energy parameters and composition-dependent peak position of $2 \mathrm{D} \mathrm{MX}_{2}$ alloy monolayers

\begin{tabular}{|c|c|c|c|c|c|}
\hline $\begin{array}{c}\text { 二维半导体 } \\
\text { 合金材料 }\end{array}$ & $E_{\mathrm{g}, 1}(\mathrm{eV})$ & $E_{\mathrm{g}, 2}(\mathrm{eV})$ & $\begin{array}{c}\text { 能带弯曲参数 } \\
(\mathrm{eV})\end{array}$ & $\begin{array}{c}\text { 特定拉曼峰的组分 } \\
\text { 依赖位移 }\left(\mathrm{cm}^{-1}\right)\end{array}$ & 备注 \\
\hline $\mathrm{Mo}_{1-x} \mathrm{~W}_{x} \mathrm{~S}_{2}$ & $1.86\left(\mathrm{MoS}_{2}\right)$ & $1.99\left(\mathrm{WS}_{2}\right)$ & 0.25 & $\omega\left(A_{1 g}\right)=401.6+16.0 x$ & 直接带隙各向同性 \\
\hline $\mathrm{Mo}_{1-x} \mathrm{~W}_{x} \mathrm{Se}_{2}$ & $1.57\left(\mathrm{MoSe}_{2}\right)$ & $1.65\left(\mathrm{WSe}_{2}\right)$ & 0.151 & $\omega\left(A_{1 \mathrm{~g}}\right)=240.1+13.7 x-5.5 x^{2}$ & 直接带隙各向同性 \\
\hline $\mathrm{MoS}_{2(1-x)} \mathrm{Se}_{2 x}$ & $1.57\left(\mathrm{MoSe}_{2}\right)$ & $1.86\left(\mathrm{MoS}_{2}\right)$ & 0.046 & $\omega\left(\mathrm{MoS}_{2}-\mathrm{E}_{2 \mathrm{~g}}{ }^{1}\right)=384.3-15.6 x(x=0-0.6)$ & 直接带隙各向同性 \\
\hline $\operatorname{ReS}_{2(1-x)} \operatorname{Se}_{2 x}$ & $1.31\left(\mathrm{ReSe}_{2}\right)$ & $1.62\left(\operatorname{ReS}_{2}\right)$ & 0.099 & $\omega\left(\operatorname{ReS}_{2}-212\right.$ peak $)=212.4-40.7 x$ & 间接带隙各向异性 \\
\hline
\end{tabular}

度比与石墨烯中的缺陷密度 $n_{\mathrm{D}}$ 直接相关, 而 $\mathrm{D}$ 峰和 $\mathrm{D}^{\prime}$ 峰的强度比则与石墨烯表面的缺陷类型有关 ${ }^{[123,124]}$. 假设石墨烯中的缺陷为一个个零维的点缺陷, 两个 缺陷之间的平均距离为 $L_{\mathrm{D}}$, 石墨烯中的缺陷密度具 有如下的关系 ${ }^{[123]}$ :

$$
n_{\mathrm{D}}\left(\mathrm{cm}^{-2}\right)=(7.3 \pm 2.2) \times 10^{9} E_{\mathrm{L}}{ }^{4}\left(I_{\mathrm{D}} / I_{\mathrm{G}}\right),
$$

式中 $I_{\mathrm{D}}$ 和 $I_{\mathrm{G}}$ 分别为 $\mathrm{D}$ 峰和 $\mathrm{G}$ 峰的强度, $E_{\mathrm{L}}$ 为人射激光的 能量. 另外, 对于由 $\mathrm{sp}^{3}$ 杂化产生的缺陷(例如氢化、氟
化等), D峰和 $\mathrm{D}^{\prime}$ 峰的强度比 $I_{\mathrm{D}} / I_{\mathrm{D}}$ 最大, 约为 13 ; 对于

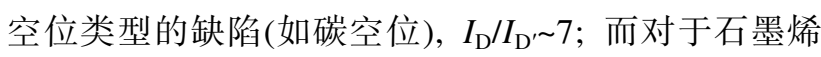
的边缘类型的缺陷, $I_{\mathrm{D}} / I_{\mathrm{D}^{\prime}}$ 最小, 约为 $3.5^{[124]}$. 据此, 可以通过拉曼光谱对石墨烯中的缺陷类型和缺陷密 度进行有效的定量化.

含有缺陷的 $\mathrm{MoS}_{2}$ 的拉曼特征也会有所不同, 图 8 (a) 所示为不同 $L_{\mathrm{D}}$ 的单层 $\mathrm{MoS}_{2}$ 的拉曼谱图, 随着 $L_{\mathrm{D}}$ 的减小, 即缺陷密度的增加, $\mathrm{E}_{2 \mathrm{~g}}$ 峰向低波数位移,
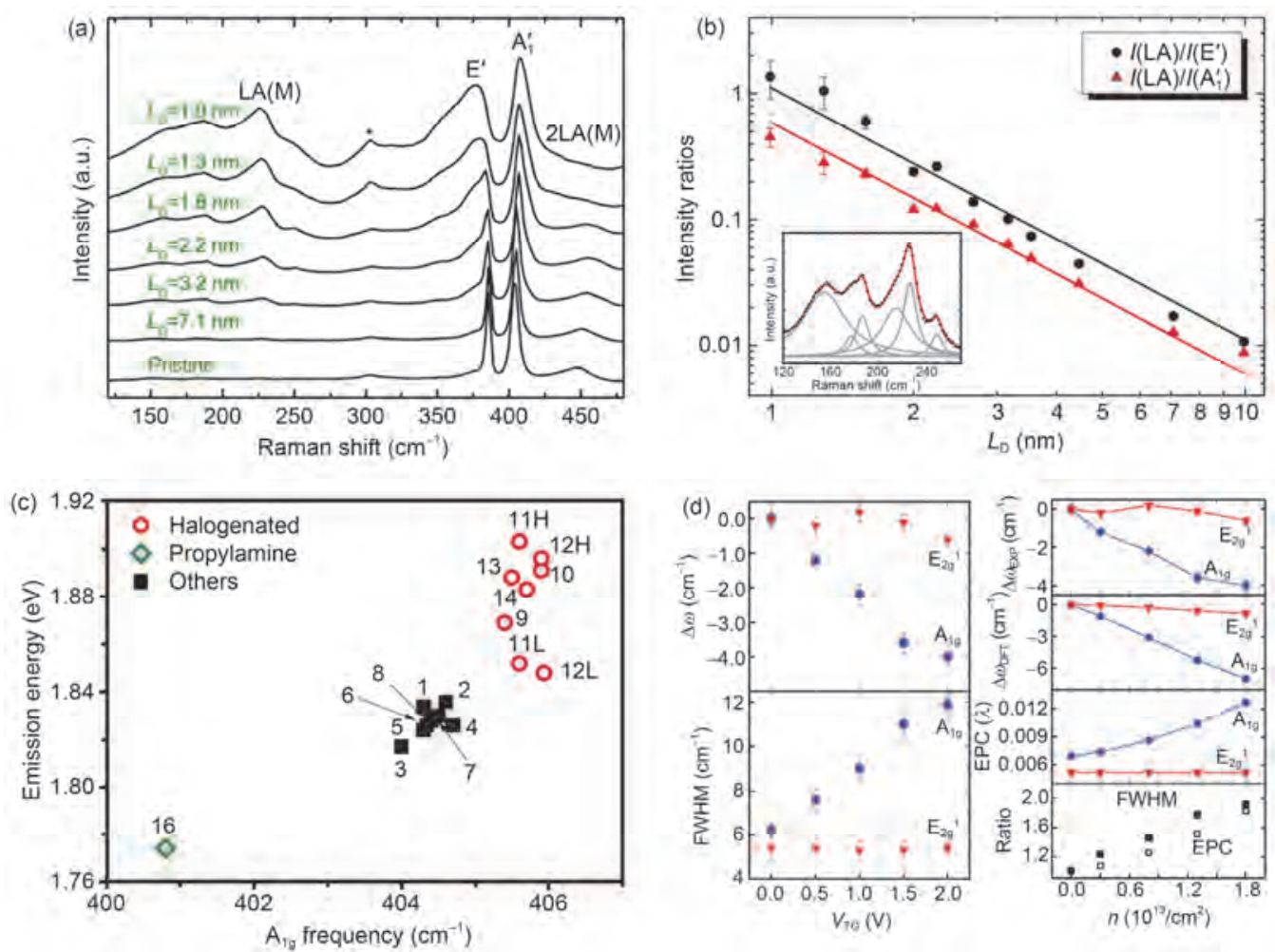

图 8 缺陷和掺杂对 $M_{0} S_{2}$ 拉曼光谱的影响. (a) 具有不同缺陷间距 $L_{\mathrm{D}}$ 的单层 $\mathrm{MoS}_{2}$ 的拉曼谱图 ${ }^{[125]}$; (b) $L A$ 声子与单层 $M_{0} S_{2}$ 的 $\mathrm{E}_{2 \mathrm{~g}}{ }^{1}$ 和 $\mathrm{A}_{1 \mathrm{~g}}$ 模的强度 比随 $L_{\mathrm{D}}$ 的变化关系 ${ }^{[125]}$; (c) 不同溶剂环境中单层 $\mathrm{MoS}_{2}$ 的苂光发射能量与 $\mathrm{A}_{1 \mathrm{~g}}$ 峰频率之间的关系 ${ }^{[126]}$; (d) 单层 $\mathrm{MoS}_{2}$ 的 $\mathrm{E}_{2 \mathrm{~g}}{ }^{1}$ 和 $\mathrm{A}_{1 \mathrm{~g}}$ 模的峰位、半峰宽 以及与电子耦合强度随㮽压和电子浓度的变化关系 ${ }^{[127]}$

Figure 8 Influence of defect and doping in Raman spectroscopy of $\mathrm{MoS}_{2}$. (a) Raman spectra of monolayer $\mathrm{MoS}_{2}$ with different interdefect distance $L_{\mathrm{D}}{ }^{[125]}$. (b) Intensity ratio $I(\mathrm{LA}) / I\left(\mathrm{E}_{2 \mathrm{~g}}{ }^{1}\right)$ and $I(\mathrm{LA}) / I\left(\mathrm{~A}_{1 \mathrm{~g}}\right)$ of monolayer $\mathrm{MoS}_{2}$ as a function of $L_{\mathrm{D}}{ }^{[125]}$. (c) Plot of photoluminescence emission energy and $\mathrm{A}_{1 \mathrm{~g}}$ frequency of monolayer $\mathrm{MoS}_{2}$ in different solvent surroundings ${ }^{[126]}$. (d) Peak position, FWHM and electron-phonon coupling of $\mathrm{E}_{2 \mathrm{~g}}{ }^{1}$ and $\mathrm{A}_{1 \mathrm{~g}}$ in monolayer $\mathrm{MoS}_{2}$ as a function of gate voltage and electron concentration ${ }^{[127]}$ 
而 $A_{1 g}$ 向高波数位移, 且 $E_{2 g}{ }^{1}$ 和 $A_{1 g}$ 峰均变宽, 密度泛 函理论(DFT)计算表明这些一阶特征峰的变化是由于 声子限域导致的 ${ }^{[125]}$. 除此之外, 在 140 420 $\mathrm{cm}^{-1}$ 的 波数范围内出现了一些新的拉曼散射峰, 这些特征 峰产生于单层 $\mathrm{MoS}_{2}$ 布里渊区边缘声子的散射, 其中 位于 $227 \mathrm{~cm}^{-1}$ 的纵向声学(LA)声子模较强, 且LA声 子模与一阶特征峰的强度比与平均缺陷间距之间存 在如下的定量关系 ${ }^{[125]}$ :

$$
\frac{I(\mathrm{LA})}{I(\mathrm{X})}=\frac{C(\mathrm{X})}{L_{\mathrm{D}}{ }^{2}},
$$

这里 $\mathrm{X}$ 代表 $\mathrm{E}_{2 \mathrm{~g}}{ }^{1}$ 或 $\mathrm{A}_{1 \mathrm{~g}}$, 其中 $C\left(\mathrm{E}_{2 \mathrm{~g}}{ }^{1}\right)=1.11 \pm 0.08 \mathrm{~nm}^{2}$, $C\left(\mathrm{~A}_{1 \mathrm{~g}}\right)=0.59 \pm 0.03 \mathrm{~nm}^{2}$. 当然, 这一关系可能与人射 激光能量相关, 目前没有相关的研究.

除了二维材料本身的晶体结构会影响其拉曼光 谱外, 周围环境的掺杂作用也会对其产生影响. 单层 石墨烯的科恩异常效应(Kohn anomaly)在电子和空 穴掺杂时均会减弱, 导致其 $\mathrm{G}$ 峰向高波数位移, 而其 $\mathrm{G}^{\prime}$ 峰在电子(空穴) 掺杂时向低波数(高波数) 位移, 石 墨烯的 $\mathrm{G}$ 峰峰位与其费米能级 $\left|E_{\mathrm{F}}\right|$ 之间存在定量关 系 ${ }^{[49]}$ :

$$
\hbar \Delta \omega=\frac{\hbar A\left\langle D_{\Gamma}^{2}\right\rangle_{\mathrm{F}}}{\pi M \omega_{0}\left(\hbar v_{\mathrm{F}}\right)^{2}}\left|\varepsilon_{\mathrm{F}}\right|=\alpha^{\prime}\left|\Delta E_{\mathrm{F}}\right|,
$$

式中 $\left\langle D_{\Gamma}^{2}\right\rangle_{\mathrm{F}}=45.6 \mathrm{eV}^{2} \AA^{-2}$ 为 $\mathrm{E}_{2 \mathrm{~g}}$ (G峰)的形变势, $M$ 为碳 原子的质量, $v_{\mathrm{F}}$ 为石墨烯的费米速度, $\omega_{0}$ 是本征石墨 烯的 $\mathrm{G}$ 峰峰位, $\alpha^{\prime}=4.39 \times 10^{-3}$.

周围环境对 $\mathrm{MoS}_{2}$ 的掺杂作用同样会引起其拉曼 特征的变化, 本课题组对单层 $\mathrm{MoS}_{2}$ 的溶剂化效应研 究发现, 将单层 $\mathrm{MoS}_{2}$ 置于丙胺中, 会对其造成电子 掺杂, 其 $\mathrm{A}_{1 \mathrm{~g}}$ 模向低波数位移, 而将其置于卤素溶剂 中, 空穴掺杂效应使得 $\mathrm{A}_{1 \mathrm{~g}}$ 模向高波数位移 ${ }^{[126]}$, 如图 8(c)所示. 另外, 对单层 $\mathrm{MoS}_{2}$ 器件的栅压调制研究发 现 ${ }^{[127]}$, 随着正栅压的增大, 即电子浓度增大, $\mathrm{MoS}_{2}$ 的 $\mathrm{A}_{1 \mathrm{~g}}$ 模向低波数位移, 且半峰宽增大, 而 $\mathrm{E}_{2 \mathrm{~g}}{ }^{1}$ 模则变 小, 电子掺杂浓度为 $1.8 \times 10^{13} \mathrm{~cm}^{-2}$ 时, $\mathrm{A}_{1 \mathrm{~g}}$ 和 $\mathrm{E}_{2 \mathrm{~g}}{ }^{1}$ 分别 向低波数位移 4 和 $0.6 \mathrm{~cm}^{-1}$, DFT计算表明, 这一现象 是由于 $\mathrm{A}_{1 \mathrm{~g}}$ 声子与电子之间的耦合强度 $(\mathrm{EPC})$ 较 $\mathrm{E}_{2 \mathrm{~g}}{ }^{1}$ 大 导致的(图8(d)).

\section{6 拉曼光谱法测定二维材料中的应变}

二维材料的拉曼特征峰对应力有着更加丰富的 响应. 石墨烯的 $\mathrm{G}$ 和 $\mathrm{G}^{\prime}$ 峰在受到拉伸应力时均向低波
数位移, 且与所受应力成线性关系, 单层石墨烯的 $\mathrm{G}$ 和 $\mathrm{G}^{\prime}$ 峰的应力系数分别为 $-14.2 \pm 0.7$ 和 $-27.8 \pm 0.8$ $\mathrm{cm}^{-1} / \%$, 这一现象是由于在拉伸应力下碳一碳键的拉 伸作用导致的, 且这一现象是可逆的, 即在释放应力 后，其拉曼特征峰将会回到未受应力时的峰位，而在 压缩应力作用下，碳原子之间的距离会变小导致 $\mathrm{G}$ 和 $\mathrm{G}^{\prime}$ 峰向高波数位移 ${ }^{[128]}$. 当施加的拉伸应力较大时, 石墨烯的 $G$ 和 $G^{\prime}$ 峰均会发生裂分, 且裂分的两个组分 均会随着应力的增大向低波数位移, 且位移系数有 所不同, 在应力施加方向与石墨烯的zigzag方向成 $11.3^{\circ}$ 夹角时, $\mathrm{G}^{+}$和 $\mathrm{G}^{-}$的应力系数分别为 -10.8 和 -31.7 $\mathrm{cm}^{-1} / \%{ }^{[106]}$; 当应力施加方向沿着zigzag方向时, $\mathrm{G}^{++}$ 和 $\mathrm{G}^{\prime-}$ 的应力系数分别为 -26.0 和 $-67.8 \mathrm{~cm}^{-1} / \%$, 而对 于 armchair方向的应力作用, 其应力系数则变为 -44.1 和 $-63.1 \mathrm{~cm}^{-1} / \%^{[107]}$.

与石墨烯不同, 单层 $\mathrm{MoS}_{2}$ 在拉伸应力作用 下 $^{[129]}$ (图9(a)和(b)), 其特征峰 $A_{1 g}$ 几乎不会发生位移, 且强度保持不变, 而 $\mathrm{E}_{2 \mathrm{~g}}{ }^{1}$ 会向低波数位移, 且会在应 力达到 $0.8 \%$ 时裂分为 $\mathrm{E}_{2 \mathrm{~g}}{ }^{1+}$ 和 $\mathrm{E}_{2 \mathrm{~g}}{ }^{1-}$ 两个峰, 这两个拉 曼峰随着应力变化的位移速率分别为 $1.0 \pm 1$ 和 $4.5 \pm 0.3$ $\mathrm{cm}^{-1} / \%$; 而在压缩应力的作用下, 单层 $\mathrm{MoS}_{2}$ 的 $\mathrm{A}_{1 \mathrm{~g}}$ 和 $\mathrm{E}_{2 \mathrm{~g}}{ }^{1}$ 则同时向高波数位移 ${ }^{[130]}$, 但是 $\mathrm{E}_{2 \mathrm{~g}}{ }^{1}$ 的位移速率约 为 $\mathrm{A}_{1 \mathrm{~g}}$ 模的 1.5 倍, 在应力大小为 $0.2 \%$ 时, $\mathrm{E}_{2 \mathrm{~g}}{ }^{1}$ 和 $\mathrm{A}_{1 \mathrm{~g}}$ 的 位移量分别为 3 和 $2 \mathrm{~cm}^{-1}$. $\mathrm{E}_{2 \mathrm{~g}}{ }^{1}$ 模对应力作用的较灵 敏的响应主要是由于 $\mathrm{E}_{2 \mathrm{~g}}{ }^{1}$ 是面内振动模, 而 $\mathrm{A}_{1 \mathrm{~g}}$ 则为 面外振动模.

黑磷各向异性的晶体结构赋予了其各向异性的 力学性能, 当应力施加方向不一致时, 其拉曼特征峰 的响应也会有所不同 ${ }^{[131]}$. 理论计算表明, 在zigzag 方向的应力作用下, $\mathrm{B}_{2 \mathrm{~g}}$ 和 $\mathrm{A}_{\mathrm{g}}{ }^{2}$ 模的频率位移是单调的, 即在拉伸应力下向低波数位移，在压缩应力下向高 波数位移, 而 $\mathrm{Ag}_{\mathrm{g}}$ 则无论在拉伸应力还是压缩应力下 均向低波数位移, 另外, 在拉伸应力下, 原本被禁阻 的 $\mathrm{B}_{3 \mathrm{~g}}{ }^{2}$ 模可以被检测到, 同时随着拉伸应力的增大略 微的向高波数位移; 在armchair方向的应力作用下, 所有的振动模均表现出单调的位移趋势, $\mathrm{B}_{2 \mathrm{~g}}$ 和 $\mathrm{A}_{\mathrm{g}}{ }^{2}$ 在 拉伸应力下向高波数位移, 在压缩应力下向低波数 位移, 而 $\mathrm{A}_{\mathrm{g}}{ }^{1}$ 则在拉伸应力下向低波数位移, 在压缩 应力下向高波数位移, 且所有的振动模的位移幅度 远小于沿着zigzag方向的应力作用的情况，这是由于 黑磷在armchair方向的褶皱的晶体结构导致的 ${ }^{[132]}$. Wang等人 ${ }^{[131]}$ 在实验上对黑磷拉曼光谱对不同方向 
(a)
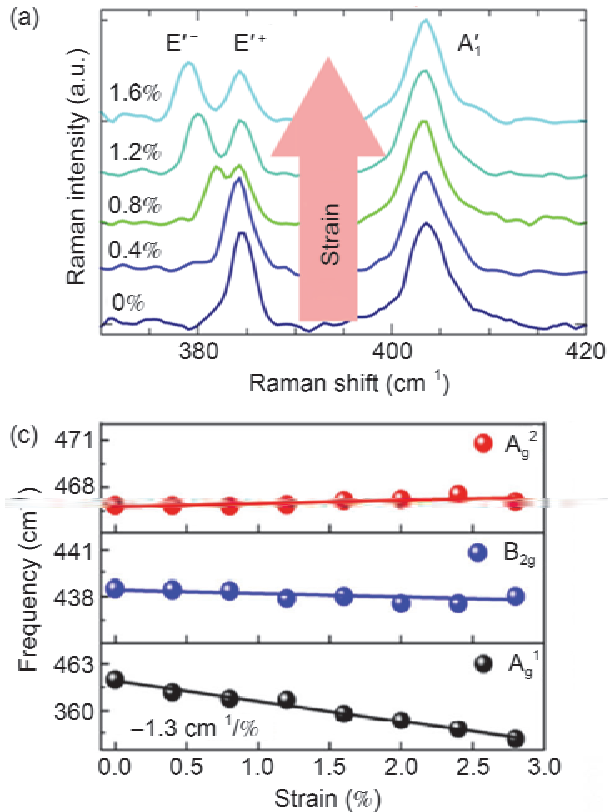
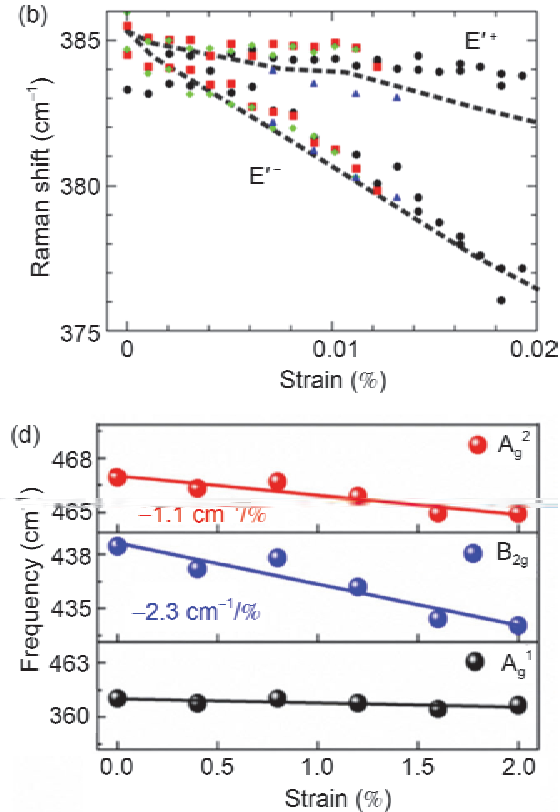

图 9 应力对二维材料拉曼光谱的影响. 拉伸应力作用下, 单层 $\mathrm{MoS}_{2}$ 的拉曼谱图(a)以及 $\mathrm{E}_{2 \mathrm{~g}}{ }^{1+}$ 和 $\mathrm{E}_{2 \mathrm{~g}}{ }^{1}{ }^{1}$ 峰位随应力的变化关系 $(\mathrm{b})^{[129]}$; 单层黑磷的 $\mathrm{A}_{\mathrm{g}}{ }^{1}, \mathrm{~B}_{2 \mathrm{~g}}$ 和 $\mathrm{A}_{\mathrm{g}}{ }^{2}$ 模在应力沿着与 armchair方向成 $15^{\circ}(\mathrm{c})$ 和与 armchair方向成 $10^{\circ}$ 夹角 (d)时的位移情况 ${ }^{[131]}$

Figure 9 Influence of strain in the Raman spectroscopy of $2 \mathrm{D}$ materials. Raman spectra (a) and $\mathrm{E}_{2 \mathrm{~g}}{ }^{1+}$ and $\mathrm{E}_{2 \mathrm{~g}}{ }^{1-}$ position (b) of monolayer MoS $\mathrm{S}_{2}$ as a function of tensile strain ${ }^{[129]}$. Plots of $\mathrm{A}_{\mathrm{g}}{ }^{1}, \mathrm{~B}_{2 \mathrm{~g}}$ and $\mathrm{A}_{\mathrm{g}}{ }^{2}$ modes position with the strain along the direction of near armchair (c) and near zigzag (d) directions ${ }^{[131]}$

应力作用的响应进行了研究, 图9((c), (d))显示, 在应 力沿着与 armchair方向成 $15^{\circ}$ 角的方向施加时, $\mathrm{A}_{\mathrm{g}}{ }^{1}$ 线 性的向低波数位移, 其位移速率为 $-1.3 \mathrm{~cm}^{-1} / \%$, 而 $\mathrm{B}_{2 \mathrm{~g}}$ 和 $\mathrm{A}_{\mathrm{g}}{ }^{2}$ 则几乎没有位移; 在应力沿着与 zigzag方向 成 $10^{\circ}$ 角的方向施加时的情况则有所不同, $\mathrm{B}_{2 \mathrm{~g}}$ 和 $\mathrm{A}_{\mathrm{g}}{ }^{2}$ 则分别以 -2.3 和 $-1.1 \mathrm{~cm}^{-1} / \%$ 的速率线性向低波数位 移, 而 $\mathrm{A}_{\mathrm{g}}{ }^{1}$ 则不受影响.

\section{7 拉曼光谱法测定二维材料的热导率}

对于具有原子级厚度的二维材料, 外界温度的 变化同样会使其拉曼特征峰发生位移, 这两者之间 通常成线性关系 ${ }^{[133]} \omega=\omega_{0}+\chi T, \chi$ 为振动模的一阶温度 系数, 表示这一振动模的峰位在温度升高 $1{ }^{\circ} \mathrm{C}$ 或者 $1 \mathrm{~K}$ 时的位移量. 在实际的拉曼测量中, 人射激光通 过光学显微镜的物镜聚焦到样品上, 使得样品的局 域温度升高, 从而反映在其拉曼峰的位移上, 通过对 不同激光功率下的拉曼峰位移进行测量, 就可以计 算出二维材料的热导率 ${ }^{[6,53,134]}$. 为了减小基底和样品 之间的热传导引起的材料热导率计算的误差, 通常 测量二维材料热导率的拉曼实验是基于悬空的样品 进行的. 同时, 通过拉曼光谱计算二维材料热导率是 基于以下两个假设条件的：(1) 激光功率的增加并不
会引起悬空的二维材料周围的基底温度的增加，即 激光引起的局域温度升高的二维材料的尺寸远小于 悬空的材料尺寸; (2) 激光聚焦到样品上产生的局域 热量是均匀地从激光斑点处向外扩散的. 在激光功 率相对较小时, 二维材料的热导率可以表示为

$$
\kappa=(1 / 2 \pi h)(\Delta P / \Delta T)=\chi(1 / 2 \pi h)(\delta \omega / \delta P)^{-1},
$$

式中, $h$ 为测量的二维材料的厚度, $\Delta T$ 为激光功率变 化 $\Delta P$ 时引起的二维材料局域温度的变化, $\delta \omega$ 为激光 功率变化 $\delta P$ 时引起的二维材料拉曼特征峰的位移. 因此, 基于二维材料拉曼峰的温度依赖性计算出其 一阶温度系数, 然后通过不同激光功率下的拉曼测 量即可计算出其热导率.

室温下悬空的单层石墨烯的热导率可以达到 (4840 5300) W/(m K), 而化学气相沉积(CVD)生长的 单层石墨烯的热导率在 350 和 $500 \mathrm{~K}$ 温度下分别为 2500和 1400 W/(m K). 与石墨烯类似, 图10((a), (b)) 可见 $\mathrm{MoS}_{2}$ 的拉曼特征峰随着激光功率的增加线性地 向低波数位移, 通过对其 $\mathrm{A}_{1 \mathrm{~g}}$ 模随激光功率的位移做 线性拟合, 可以计算得知 $6.5 \mathrm{~nm}$ 厚的 $\mathrm{MoS}_{2}$ 的热导率约 为 $52 \mathrm{~W} /(\mathrm{m} \mathrm{K})^{[134]}$. 具有各向异性结构的黑磷同样具 有各向异性的热导率，理论计算表明，黑磷zigzag和 armchair方向的热导率分别为 48.9 和 $27.8 \mathrm{~W} /(\mathrm{m} \mathrm{K})^{[136]}$, 

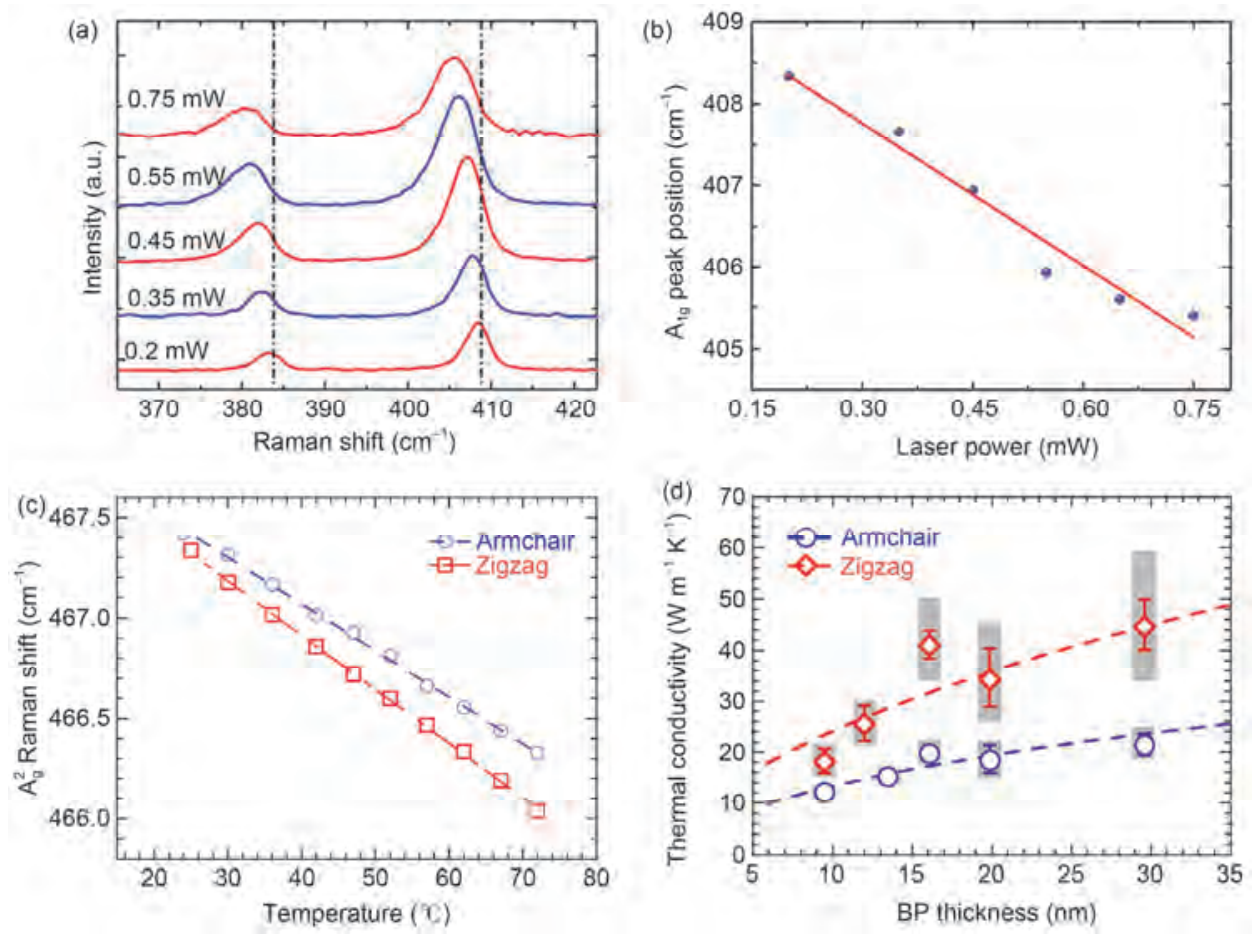

图 10 二维材料拉曼光谱的热效应. 不同功率的激光激发下, 少层 $\mathrm{MoS}_{2}$ 的拉曼谱图(a)和 $\mathrm{A}_{1 \mathrm{~g}}$ 模(b)的峰位 ${ }^{[134]} ;(\mathrm{c}) 9.5 \mathrm{~nm}$ 厚黑磷的 $\mathrm{A}_{\mathrm{g}}{ }^{2}$ 峰位随温 度的变化关系 ${ }^{[135]} ;$ (d) 黑磷zigzag和armchair方向的热导率与样品厚度的关系 ${ }^{[135]}$

Figure 10 Thermal effect of Raman spectroscopy of 2D materials. Raman spectra (a) and $\mathrm{A}_{1 \mathrm{~g}}$ frequency (b) of fewlayer MoS $\mathrm{S}_{2}$ as a function of laser power ${ }^{[134]}$. (c) $\mathrm{A}_{\mathrm{g}}{ }^{2}$ peak position of a black phosphorus sheet with $9.5 \mathrm{~nm}$ thickness as a function of temperature ${ }^{[135]}$. (d) Plot of zigzag and armchair in-plane thermal conductivity of black phosphorus with different thickness ${ }^{[135]}$

Luo等人 ${ }^{[135]}$ 通过拉曼光谱测量黑磷各向异性的热导 率(图10(c)和(d)), 在黑磷样品的厚度大于 $15 \mathrm{~nm}$ 时, 其 armchair和zigzag方向的热导率分别为 20和 40 W/(m $\mathrm{K})$, 而其热导率随着厚度的减小而减小到 10 和 20 $\mathrm{W} /(\mathrm{m} \mathrm{K})$, 表现出了显著的各向异性, 这一各向异性 与黑磷的各向异性的声子色散相关.

\section{8 拉曼光谱法测定二维电荷密度波材料的 相变}

电荷密度波 $(\mathrm{CDW})$ 是形成驻波状电荷密度的一 种现象, 通常伴随着电子-声子相互作用引起的晶格 变形和带隙的打开. 第五副族的过渡金属二硫族化 合物 $\mathrm{MX}_{2}(\mathrm{M}=\mathrm{V}, \mathrm{Nb}, \mathrm{Ta} ; \mathrm{X}=\mathrm{S}, \mathrm{Se})$ 由于具有 $\mathrm{CDW}$ 特性 而备受瞩目, 相关块体材料的 CDW特性在 20 世纪就 已经被广泛研究, 例如, $1 \mathrm{~T}-\mathrm{TaS}_{2}$ 具有 $\mathrm{CDW}$ 特性, 其 正常的金属相与非公度结构 (ICCDW) 的相变温度约 为 $550 \mathrm{~K}$, 非公度结构与近公度结构 (NCCDW) 的相 变温度约为 $350 \mathrm{~K}$, 近公度结构与公度结构 (CCDW) 的相变温度约为 $180 \mathrm{~K}$, 在 $180 \mathrm{~K}$ 以下, 所有的 $\mathrm{Ta}$ 原
子会形成 $\sqrt{13} \times \sqrt{13}$ 的David star超结构, 每一个David $\operatorname{star}$ 结构包含有 13 个 $\mathrm{Ta}$ 原子 ${ }^{[137,138]}$ (图 11(a)). 此外, $2 \mathrm{H}-\mathrm{TaS}_{2}$ 的 $\mathrm{CDW}$ 相变温度约为 $T_{\mathrm{CDW}, \mathrm{C}} \sim 75 \mathrm{~K}^{[139]}$; $1 \mathrm{~T}-\mathrm{TaSe}_{2}$ 的 $\mathrm{CDW}$ 相变温度约为 $T_{\mathrm{CDW}, \mathrm{C}} \sim 473 \mathrm{~K}$ 和 $T_{\mathrm{CDW}, \mathrm{IC}} \sim 600 \mathrm{~K}^{[140]} ; 2 \mathrm{H}-\mathrm{TaSe}_{2}$ 的 $\mathrm{CDW}$ 相变温度约为 $T_{\mathrm{CDW}, \mathrm{C}} \sim 90 \mathrm{~K}$ 和 $T_{\mathrm{CDW}, \mathrm{IC}} \sim 123 \mathrm{~K}^{[81]} ; 2 \mathrm{H}-\mathrm{NbSe}_{2}$ 的 CDW相 变温度为 $T_{\mathrm{CDW}} \sim 35 \mathrm{~K}^{[141]} ; 1 \mathrm{~T}-\mathrm{VS}_{2}$ 和 $1 \mathrm{~T}-\mathrm{VSe}_{2}$ 的 $\mathrm{CDW}$ 相 变温度分别为 $305^{[142]}$ 和 $107 \mathrm{~K}^{[143]}$.

$\mathrm{CDW}$ 材料在相变过程中, 结构的对称性必然会 发生变化, 使得其拉曼特征发生显著的变化, 因此拉 曼光谱同样可以用来研究 $\mathrm{CDW}$ 相变材料的相变特 性 ${ }^{[138,144,145]}$. 以 $1 \mathrm{~T}-\mathrm{TaS}_{2}$ 为例, 其正常的金属相属于 $\mathrm{D}_{3 \mathrm{~d}}$ 点群，拉曼活性的振动模具有 $\mathrm{A}_{1 \mathrm{~g}}$ 和 $\mathrm{E}_{\mathrm{g}}$ 对称性，而 $\mathrm{CCDW}$ 相属于三斜晶系的 $\mathrm{C}_{\mathrm{i}}$ 点群 ${ }^{[146]}$, 仅 $\mathrm{A}_{\mathrm{g}}$ 模具有拉 曼活性, 其拉曼谱图必然会有相应的变化.

图11((b), (c))为单层和6.8 $\mathrm{nm}$ 厚的 $1 \mathrm{~T}-\mathrm{TaS}_{2}$ 在 4 300 K温度范围内升温过程的二维拉曼成像, 对应 的温度以来的拉曼峰位示于图11((d), (e))中. 可见升 温过程中, 所有的拉曼特征峰在 $230 \mathrm{~K}$ 以上向低波 

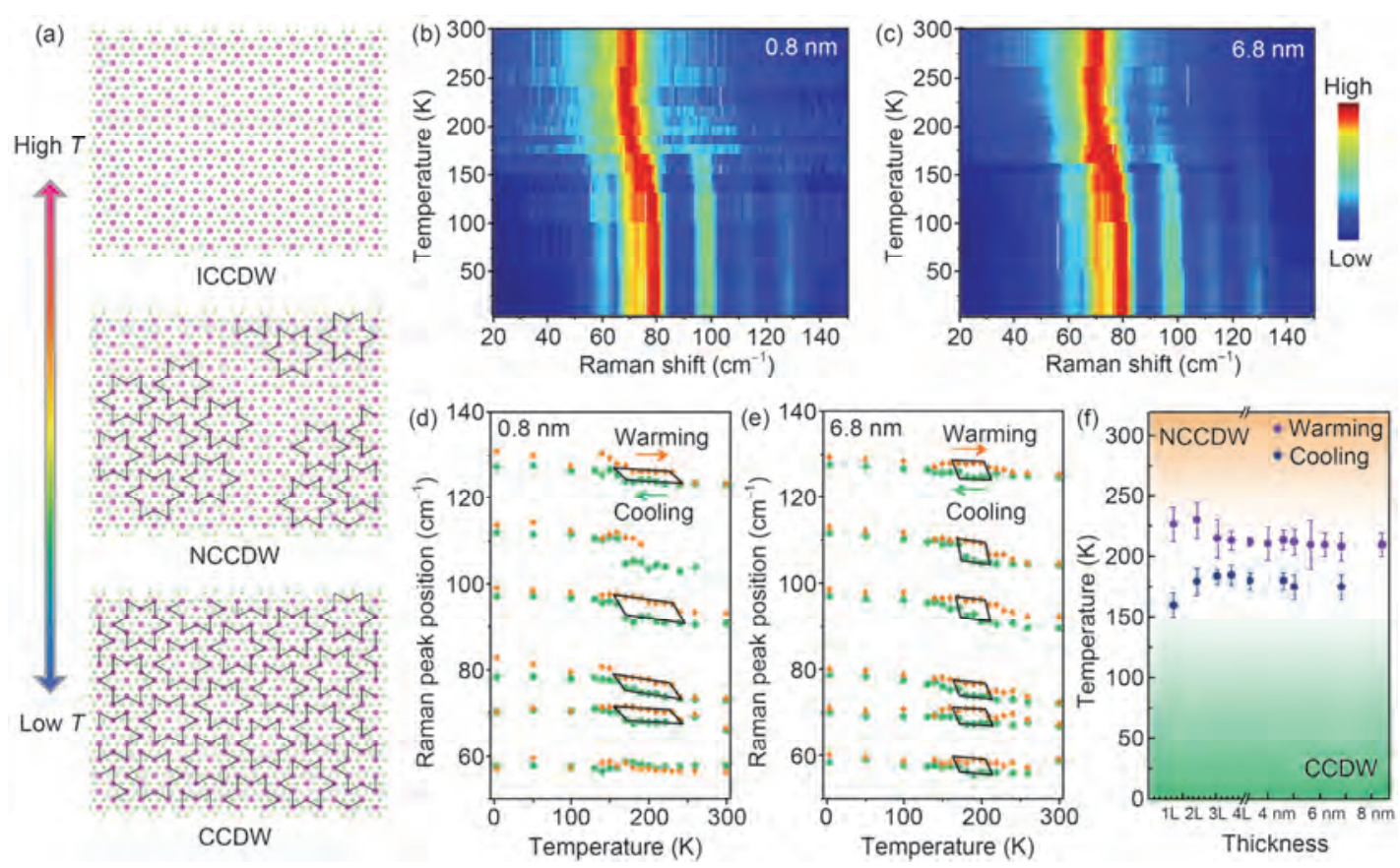

图 $111 \mathrm{~T}-\mathrm{TaS}_{2} \mathrm{CDW}$ 相变过程的拉曼光谱原位监测 ${ }^{[137]}$. (a) $1 \mathrm{~T}-\mathrm{TaS}_{2}$ 结构的 $\mathrm{CDW}$ 相变示意图; 单层(b)和 $6.8 \mathrm{~nm}(\mathrm{c})$ 厚的 $1 \mathrm{~T}-\mathrm{TaS}_{2}$ 样品在 4 300 K 温度范围内的二维拉曼成像(升温过程); 单层(d)和6.8 nm(e)厚的 $1 \mathrm{~T}^{-} \mathrm{TaS}_{2}$ 拉曼特征峰位移随温度的变化关系(升温和降温过程); (f) $1 \mathrm{~T}-\mathrm{TaS}_{2}$ 的 $\mathrm{CDW}$ 相变温度的厚度依赖性

Figure 11 In-situ probing the CDW phase transition of $1 \mathrm{~T}-\mathrm{TaS}_{2}$ by Raman spectroscopy ${ }^{[137]}$. (a) Schematic of $1 \mathrm{~T}-\mathrm{TaS}_{2}$ structural CDW phase transition. Two dimensional Raman spectra of $1 \mathrm{~T}-\mathrm{TaS}_{2}$ (b) monolayer and (c) thicker $(6.8 \mathrm{~nm}$ ) sheets (warming process). Raman peak frequency of 1T-TaS (d) monolayer and (e) thicker sheets as a function of temperature. (f) Thickness-dependent CDW phase transition temperature

数位移, 而在降温过程中拉曼峰向高波数位移的温 度约为 $160 \mathrm{~K}$, 另外, 位于 75 和 $83 \mathrm{~cm}^{-1}$ 的两个特征 峰峰位在高温部分更加接近 ${ }^{[137]}$, 这是由于 $\mathrm{CCDW}$ 相 声子布里渊区的折叠导致的, 这一特征同时也是 $1 \mathrm{~T}-\mathrm{TaS}_{2}$ 的 CCDW-NCCDW 相变的直接证据, 且 CCDW-NCCDW 和NCCDW-CCDW相变过程中的不 同势垒导致升温过程的相变温度较降温过程中高, 形成了相变回滞窗口 ${ }^{[144]}$. 不同厚度的 $1 \mathrm{~T}-\mathrm{TaS}_{2}$ 变温 拉曼研究发现(图11(f)), 随着厚度的减小, 升温过程 的相变温度稍有增加, 而降温过程的相变温度则有 所降低 ${ }^{[137]}$. 这可能与不同相的本征层数依赖的稳定 性相关, 同时, 由于薄层样品成核区域增强的牵制作 用引起了较大的活化势垒 ${ }^{[144]}$, 使得相变回滞窗口随 着 $1 \mathrm{~T}-\mathrm{TaS}_{2}$ 层数的减小而变大. 同样的, 拉曼光谱也可 以用来测定其他的二维 $\mathrm{CDW}$ 相变材料的相变特性, 已有文献报道了 $1 \mathrm{~T}-\mathrm{TaSe}_{2}{ }^{[54]}, 1 \mathrm{~T}-\mathrm{VS}_{2}$ 和 $1 \mathrm{~T}-\mathrm{VSe}_{2}{ }^{[147]}$ 的 $\mathrm{CDW}$ 相变过程的拉曼特征峰变化.

\section{9 总结和展望}

本文介绍了二维材料的基本拉曼特征及其结构
依赖性. 通过对二维材料拉曼光谱的测量，可以根据 其特征峰的峰位、峰强以及峰形的变化快速准确地确 定其层数. 在二维材料的边缘处, 其结构对称性的降 低会破坏其常规的拉曼选律，据此可以对其边缘手 性进行指认，根据二维材料拉曼特征峰强度的晶格 方向依赖性, 可以判定其晶格方向.二维 $\mathrm{MX}_{2}$ 合金成 分的变化会引起其相应拉曼特征峰的位移, 结合 MREI模型对其拟合，不仅可以对未知的合金组分进 行定量化, 还可以获得二维材料原子振动的力常数. 缺陷的存在会导致二维材料新的拉曼振动模的出现, 根据其与二维材料的典型拉曼特征峰的强度比可以 测定二维材料的缺陷密度. 掺杂、外界应力以及热效 应均会引起二维材料拉曼特征峰的位移, 结合二维 材料的电子能带结构, 可以定量研究二维材料的掺 杂浓度、应力以及热导率. 另外, 二维电荷密度波材 料相变过程中的结构变化同样会引起其拉曼选律的 改变, 据此可以通过拉曼光谱研究其相变特性.

二维材料拉曼光谱的研究还有很长的路要走, 还会遇到很多科学和技术上的难题. 比如, 在分析二 维材料的拉曼选律时, 其拉曼张量的矩阵元与材料 
的层数以及人射激光的波长之间的关系; 光在二维 材料中的传播对其拉曼特征峰的影响; 在同时考虑 各向异性二维材料的各向异性吸收和双折射效应, 其晶格方向依赖的拉曼峰强与样品层数以及激光波
长之间的对应关系等等. 总之, 拉曼光谱在二维材料 的研究中还有非常广阔的应用前景, 相信随着科学 家们的深人研究, 这些问题终会被一一解决, 从而为 二维材料的可控制备与器件应用提供支撑.

\section{参考文献}

1 Novoselov K S, Geim A K, Morozov S V, et al. Electric field effect in atomically thin carbon films. Science, 2004, 306: 666-669

2 Novoselov K S, Geim A K, Morozov S V, et al. Two-dimensional gas of massless dirac fermions in graphene. Nature, 2005, 438: 197-200

3 Novoselov K S, Jiang Z, Zhang Y, et al. Room-temperature quantum hall effect in graphene. Science, 2007, $315: 1379$

4 Du X, Skachko I, Duerr F, et al. Fractional quantum hall effect and insulating phase of dirac electrons in graphene. Nature, 2009, 462: 192-195

5 Nair R R, Blake P, Grigorenko A N, et al. Fine structure constant defines visual transparency of graphene. Science, 2008, 320: $1308-1308$

6 Balandin A A, Ghosh S, Bao W Z, et al. Superior thermal conductivity of single-layer graphene. Nano Lett, 2008, 8: 902-907

7 Dean C R, Young A F, Meric I, et al. Boron nitride substrates for high-quality graphene electronics. Nat Nanotechnol, 2010, 5: 722-726

8 Geim A K, Novoselov K S. The rise of graphene. Nat Mater, 2007, 6: 183-191

9 Akinwande D, Petrone N, Hone J. Two-dimensional flexible nanoelectronics. Nat Commun, 2014, 5: 5678

10 Bae S, Kim H, Lee Y, et al. Roll-to-roll production of 30-inch graphene films for transparent electrodes. Nat Nanotechnol, 2010, 5: $574-578$

11 Mueller T, Xia F N A, Avouris P. Graphene photodetectors for high-speed optical communications. Nat Photonics, 2010, 4: 297-301

12 Zhang Y B, Tang T T, Girit C, et al. Direct observation of a widely tunable bandgap in bilayer graphene. Nature, 2009, 459: 820-823

13 Zhang L M, Yu J W, Yang M M, et al. Janus graphene from asymmetric two-dimensional chemistry. Nat Commun, 2013, 4: 1443

14 Zhang C H, Fu L, Liu N, et al. Synthesis of nitrogen-doped graphene using embedded carbon and nitrogen sources. Adv Mater, 2011, 23: $1020-1024$

15 Balog R, Jorgensen B, Nilsson L, et al. Bandgap opening in graphene induced by patterned hydrogen adsorption. Nat Mater, 2010, 9: 315-319

16 Wu J, Xie L M, Li Y G, et al. Controlled chlorine plasma reaction for noninvasive graphene doping. J Am Chem Soc, 2011, 133: 19668-19671

17 Robinson J T, Burgess J S, Junkermeier C E, et al. Properties of fluorinated graphene films. Nano Lett, 2010, 10: 3001-3005

18 Radisavljevic B, Radenovic A, Brivio J, et al. Single-layer $\mathrm{MoS}_{2}$ transistors. Nat Nanotechnol, 2011, 6: 147-150

19 Lee H S, Min S W, Chang Y G, et al. $\mathrm{MoS}_{2}$ nanosheet phototransistors with thickness-modulated optical energy gap. Nano Lett, 2012, 12: $3695-3700$

20 Xie L M. Two-dimensional transition metal dichalcogenide alloys: Preparation, characterization and applications. Nanoscale, 2015, 7: $18392-18401$

21 Kubota Y, Watanabe K, Tsuda O, et al. Deep ultraviolet light-emitting hexagonal boron nitride synthesized at atmospheric pressure. Science, 2007, 317: 932-934

22 Tongay S, Zhou J, Ataca C, et al. Thermally driven crossover from indirect toward direct bandgap in 2D semiconductors: MoSe ${ }_{2}$ versus $\mathrm{MoS}_{2}$. Nano Lett, 2012, 12: 5576-5580

23 Gutierrez H R, Perea-Lopez N, Elias A L, et al. Extraordinary room-temperature photoluminescence in triangular $\mathrm{WS}_{2}$ monolayers. Nano Lett, 2013, 13: 3447-3454

24 Chiritescu C, Cahill D G, Nguyen N, et al. Ultralow thermal conductivity in disordered, layered WSe ${ }_{2}$ crystals. Science, 2007, 315: 351-353

25 Zhang M, Zhu Y M, Wang X S, et al. Controlled synthesis of $\mathrm{ZrS}_{2}$ monolayer and few layers on hexagonal boron nitride. J Am Chem Soc, 2015, 137: 7051-7054

26 Yu Y J, Yang F Y, Lu X F, et al. Gate-tunable phase transitions in thin flakes of 1T-TaS 2 . Nat Nanotechnol, 2015, 10: 270-276

27 Tongay S, Sahin H, Ko C, et al. Monolayer behaviour in bulk $\mathrm{ReS}_{2}$ due to electronic and vibrational decoupling. Nat Commun, 2014, 5: 3252

28 Lei S D, Ge L H, Liu Z, et al. Synthesis and photoresponse of large GaSe atomic layers. Nano Lett, 2013, 13: 2777-2781 
29 Hu P G, Zhang J, Yoon M N, et al. Highly sensitive phototransistors based on two-dimensional GaTe nanosheets with direct bandgap. Nano Res, 2014, 7: 694-703

30 Li L, Chen Z, Hu Y, et al. Single-layer single-crystalline SnSe nanosheets. J Am Chem Soc, 2013, 135: 1213-1216

31 Sriv T, Kim K, Cheong H. Low-frequency Raman spectroscopy of few-layer 2H-SnS 2 . Sci Rep, 2018, 8: 10194

32 Gonzalez J M, Oleynik I I. Layer-dependent properties of $\mathrm{SnS}_{2}$ and $\mathrm{SnSe}_{2}$ novel two-dimensional materials. Phys Rev B, 2016, 94: 125443

33 Xia F N, Wang H, Jia Y C. Rediscovering black phosphorus as an anisotropic layered material for optoelectronics and electronics. Nat Commun, 2014, 5: 4458

34 Vogt P, De Padova P, Quaresima C, et al. Silicene: Compelling experimental evidence for graphene like two-dimensional silicon. Phys Rev Lett, 2012, 108: 155501

35 Li L F, Lu S Z, Pan J B, et al. Buckled germanene formation on Pt(111). Adv Mater, 2014, 26: 4820-4824

36 Chhowalla M, Shin H S, Eda G, et al. The chemistry of two-dimensional layered transition metal dichalcogenide nanosheets. Nat Chem, 2013, 5: 263-275

37 Geim A K, Grigorieva I V. Van der waals heterostructures. Nature, 2013, 499: 419-425

38 Zhang Y B, Tan Y W, Stormer H L, et al. Experimental observation of the quantum hall effect and berry's phase in graphene. Nature, 2005, 438: 201-204

39 Britnell L, Ribeiro R M, Eckmann A, et al. Strong light-matter interactions in heterostructures of atomically thin films. Science, 2013, 340: $1311-1314$

40 Splendiani A, Sun L, Zhang Y B, et al. Emerging photoluminescence in monolayer $\mathrm{MoS}_{2}$. Nano Lett, 2010, 10: 1271-1275

41 Li H, Wu J M T, Huang X, et al. Rapid and reliable thickness identification of two-dimensional nanosheets using optical microscopy. ACS Nano, 2013, 7: 10344-10353

42 Blake P, Hill E W, Castro Neto A H, et al. Making graphene visible. Appl Phys Lett, 2007, 91: 063124

43 Yu Q K, Jauregui L A, Wu W, et al. Control and characterization of individual grains and grain boundaries in graphene grown by chemical vapour deposition. Nat Mater, 2011, 10: 443-449

44 Huang P Y, Ruiz-Vargas C S, van der Zande A M, et al. Grains and grain boundaries in single-layer graphene atomic patchwork quilts. Nature, 2011, 469: 389-392

45 Wang Y Y, Gao R X, Ni Z H, et al. Thickness identification of two-dimensional materials by optical imaging. Nanotechnology, 2012, 23: 495713

46 Ni Z H, Wang Y Y, Yu T, et al. Raman spectroscopy and imaging of graphene. Nano Res, 2008, 1: 273-291

47 Casiraghi C, Hartschuh A, Qian H, et al. Raman spectroscopy of graphene edges. Nano Lett, 2009, 9: 1433-1441

48 Zhang S, Yang J, Xu R J, et al. Extraordinary photoluminescence and strong temperature/angle-dependent Raman responses in few-layer phosphorene. ACS Nano, 2014, 8: 9590-9596

49 Yan J, Zhang Y B, Kim P, et al. Electric field effect tuning of electron-phonon coupling in graphene. Phys Rev Lett, 2007, 98 : 166802

50 Pisana S, Lazzeri M, Casiraghi C, et al. Breakdown of the adiabatic born-oppenheimer approximation in graphene. Nat Mater, 2007, 6: 198-201

51 Dumcenco D O, Chen K Y, Wang Y P, et al. Raman study of 2H-Mo $\mathrm{oo}_{1-x} \mathrm{~W}_{x} \mathrm{~S}_{2}$ layered mixed crystals. J Alloy Compd, 2010, 506: 940-943

52 Zhang S Q, Mao N N, Wu J X, et al. In-plane uniaxial strain in black phosphorus enables the identification of crystalline orientation. Small, 2017, 13: 1700466

53 Cai W W, Moore A L, Zhu Y W, et al. Thermal transport in suspended and supported monolayer graphene grown by chemical vapor deposition. Nano Lett, 2010, 10: 1645-1651

54 Samnakay R, Wickramaratne D, Pope T R, et al. Zone-folded phonons and the commensurate-incommensurate charge-density-wave transition in $1 \mathrm{~T}-\mathrm{TaSe}_{2}$ thin films. Nano Lett, 2015, 15: 2965-2973

55 Ling X, Xie L M, Fang Y, et al. Can graphene be used as a substrate for Raman enhancement? Nano Lett, 2010, 10: 553-561

56 Sugai S, Ueda T, Murase K. Pressure-dependence of the lattice vibration in the orthorhombic and rhombohedral structures of black phosphorus. J Phys Soc Jpn, 1981, 50: 3356-3361

57 Wang Q H, Kalantar-Zadeh K, Kis A, et al. Electronics and optoelectronics of two-dimensional transition metal dichalcogenides. Nat Nanotechnol, 2012, 7: 699-712

58 Qian X F, Liu J W, Fu L, et al. Quantum spin hall effect in two-dimensional transition metal dichalcogenides. Science, 2014, 346: $1344-1347$

59 Lu X, Luo X, Zhang J, et al. Lattice vibrations and Raman scattering in two-dimensional layered materials beyond graphene. Nano Res, 2016, 9: 3559-3597 
60 Ferrari A C, Basko D M. Raman spectroscopy as a versatile tool for studying the properties of graphene. Nat Nanotechnol, 2013, 8: $235-246$

61 Islam M S, Ushida K, Tanaka S, et al. Analysis of vibrational properties of C-doped hexagonal boron nitride (h-BN). Comp Mater Sci, 2014, 94: 225-233 Gorbachev R V, Riaz I, Nair R R, et al. Hunting for monolayer boron nitride: Optical and Raman signatures. Small, 2011, 7: 465-468 Qiao J S, Kong X H, Hu Z X, et al. High-mobility transport anisotropy and linear dichroism in few-layer black phosphorus. Nat Commun, 2014, 5: 4475

Lu W L, Nan H Y, Hong J H, et al. Plasma-assisted fabrication of monolayer phosphorene and its Raman characterization. Nano Res, 2014, 7: 853-859

Chanchal, Garg A K. Mrei-model calculations of Raman-active modes in layered mixed crystals $\operatorname{TiS}_{2-x} \mathrm{Se}_{x}(0 \leqslant x \leqslant 2)$. J Raman Spectrosc, 2008, 39: 115-118

Cui L, He R, Li G M, et al. Raman spectroscopy of optical phonon and charge density wave modes in 1T-TiSe $e_{2}$ exfoliated flakes. Solid State Commun, 2017, 266: 21-25

70 Tsai H S, Liou J W, Setiyawati I, et al. Photoluminescence characteristics of multilayer $\mathrm{HfSe}_{2}$ synthesized on sapphire using ion implantation. Adv Mater Interfaces, 2018, 5: 1701619

71 Cingolani A, Lugara M, Levy F. Resonance Raman-scattering in $\mathrm{HfSe}_{2}$ and $\mathrm{HfS}_{2}$. Phys Scripta, 1988, 37: 389-391

72 Yuan J T, Wu J J, Hardy W J, et al. Facile synthesis of single crystal vanadium disulfide nanosheets by chemical vapor deposition for efficient hydrogen evolution reaction. Adv Mater, 2015, 27: 5605-5609

73 Yang C, Feng J R, Lv F, et al. Metallic graphene-like $\mathrm{VSe}_{2}$ ultrathin nanosheets: Superior potassium-ion storage and their working mechanism. Adv Mater, 2018, 30: 1800036

74 Sugai S, Murase K, Uchida S, et al. Investigation of the charge density waves in $1 \mathrm{~T}-\mathrm{VSe}_{2}$ by Raman scattering. J Phys Colloq, 1981, 42: $740-742$

75 Sofer Z, Sedmidubsky D, Luxa J, et al. Universal method for large-scale synthesis of layered transition metal dichalcogenides. Chem-Eur J, 2017, 23: 10177-10186

76 Mcmullan W G, Irwin J C. Raman-scattering from 2H-NbS 2 and 3R-NbS 2 . Solid State Commun, 1983, 45: 557-560

77 Wang X S, Lin J H, Zhu Y M, et al. Chemical vapor deposition of trigonal prismatic $\mathrm{NbS}_{2}$ monolayers and 3R-polytype few-layers. Nanoscale, 2017, 9: 16607-16611

78 Wang C S, Chen J M. Raman-spectrum of metallic layered compound $\mathrm{NbSe}_{2}$. Solid State Commun, 1974, 14: 1145-1148

79 Chen H X, Li Z L, Fan X, et al. Quantum linear magnetoresistance in NbTe 2 . Solid State Commun, 2018, 275: 16-20

80 Erdogan H, Kirby R D. Raman-spectrum and lattice-dynamics of $\mathrm{NbTe}_{2}$. Solid State Commun, 1989, 70: 713-715

81 Yan J A, Dela Cruz M A, Cook B, et al. Structural, electronic and vibrational properties of few-layer 2H-and 1T-TaSe 2 . Sci Rep, 2015, 5: 16646

82 Feng Q L, Zhu Y M, Hong J H, et al. Growth of large-area 2D $\operatorname{MoS}_{2(1-x)} \mathrm{se}_{2 x}$ semiconductor alloys. Adv Mater, 2014, 26: 2648-2653

83 Zhao Y Y, Luo X, Li H, et al. Interlayer breathing and shear modes in few-trilayer $\mathrm{MoS}_{2}$ and $\mathrm{WSe}_{2}$. Nano Lett, 2013, 13: 1007-1015

84 Zhang M, Wu J X, Zhu Y M, et al. Two-dimensional molybdenum tungsten diselenide alloys: Photoluminescence, Raman scattering, and electrical transport. ACS Nano, 2014, 8: 7130-7137

85 Caramazza S, Collina A, Stellino E, et al. First- and second-order Raman scattering from $\mathrm{MoTe}_{2}$ single crystal. Eur Phys J B, 2018, 91 : 35

86 Chen Y F, Xi J Y, Dumcenco D O, et al. Tunable band gap photoluminescence from atomically thin transition-metal dichalcogenide alloys. ACS Nano, 2013, 7: 4610-4616

87 Chen Y F, Dumcenco D O, Zhu Y M, et al. Composition-dependent Raman modes of $\mathrm{Mo}_{1-x} \mathrm{~W}_{x} \mathrm{~S}_{2}$ monolayer alloys Nanoscale, 2014, 4: 2833-2839

88 Song Q J, Pan X C, Wang H F, et al. The in-plane anisotropy of $\mathrm{WTe}_{2}$ investigated by angle-dependent and polarized Raman spectroscopy. Sci Rep, 2016, 6: 29254

89 Wen W, Zhu Y M, Liu X L, et al. Anisotropic spectroscopy and electrical properties of $2 \mathrm{D} \operatorname{ReS}_{2(1-x)} \operatorname{Se}_{2 x}$ alloys with distorted 1T structure. Small, 2017, 13: 1603788

90 Rybkovskiy D V, Arutyunyan N R, Orekhov A S, et al. Size-induced effects in gallium selenide electronic structure: The influence of interlayer interactions. Phys Rev B, 2011, 84: 085314 
91 Kuroda N, Ueno O, Nishina Y. Lattice-dynamical and photoelastic properties of GaSe under high-pressures studied by Raman-scattering and electronic susceptibility. Phys Rev B, 1987, 35: 3860-3870

92 Zhao S L, Wang H A, Zhou Y, et al. Controlled synthesis of single-crystal SnSe nanoplates. Nano Res, 2015, 8: 288-295

93 Nikolic P M, Milkovic L, Mihajlovic P, et al. Raman-scattering in SnSe. Czech J Phys, 1978, 28: 456-459

94 Chandrasekhar H R, Humphreys R G, Zwick U, et al. Infrared and Raman spectra of the IV-VI compounds SnS and SnSe. Phys Rev B, 1977, 15: 2177-2183

95 Wang Y Y, Ni Z H, Shen Z X, et al. Interference enhancement of Raman signal of graphene. Appl Phys Lett, 2008, 92 : 043121

96 Gupta A, Chen G, Joshi P, et al. Raman scattering from high-frequency phonons in supported n-graphene layer films. Nano Lett, 2006, 6: $2667-2673$

97 Malard L M, Pimenta M A, Dresselhaus G, et al. Raman spectroscopy in graphene. Phys Rep, 2009, 473: 51-87

98 Lee C, Yan H, Brus L E, et al. Anomalous lattice vibrations of single- and few-layer MoS 2 . ACS Nano, 2010, 4: 2695-2700

99 Li S L, Miyazaki H, Song H, et al. Quantitative Raman spectrum and reliable thickness identification for atomic layers on insulating substrates. ACS Nano, 2012, 6: 7381-7388

100 Li H, Zhang Q, Yap C C R, et al. From bulk to monolayer MoS 2 : Evolution of Raman scattering. Adv Funct Mater, 2012, 22: 1385-1390

101 Wang X M, Jones A M, Seyler K L, et al. Highly anisotropic and robust excitons in monolayer black phosphorus. Nat Nanotechnol, 2015, 10: $517-521$

102 You Y M, Ni Z H, Yu T, et al. Edge chirality determination of graphene by Raman spectroscopy. Appl Phys Lett, 2008, 93 : 163112

$103 \mathrm{Wu} \mathrm{J}$ X, Xu H, Mu W H, et al. Observation of low-frequency combination and overtone Raman modes in misoriented graphene. J Phys Chem C, 2014, 118: 3636-3643

104 Wu J X, Xu H, Zhang J. Raman spectroscopy of graphene (in Chinese). Acta Chim Sin, 2014, 72: 301-318 [吴娟霞，徐华，张锦. 拉曼 光谱在石墨烯结构表征中的应用. 化学学报, 2014, 72: 301-318]

105 Ribeiro H B, Villegas C E P, Bahamon D A, et al. Edge phonons in black phosphorus. Nat Commun, 2016, 7: 12191

106 Mohiuddin T M G, Lombardo A, Nair R R, et al. Uniaxial strain in graphene by Raman spectroscopy: G peak splitting, gruneisen parameters, and sample orientation. Phys Rev B, 2009, 79: 205433

107 Yoon D, Son Y W, Cheong H. Strain-dependent splitting of the double-resonance Raman scattering band in graphene. Phys Rev Lett, 2011, 106: 155502

108 Wang Y L, Cong C X, Qiu C Y, et al. Raman spectroscopy study of lattice vibration and crystallographic orientation of monolayer MoS 2 under uniaxial strain. Small, 2013, 9: 2857-2861

109 Wu J X, Mao N N, Xie L M, et al. Identifying the crystalline orientation of black phosphorus using angle-resolved polarized Raman spectroscopy. Angew Chem Int Ed, 2015, 54: 2366-2369

110 Sugai S, Shirotani I. Raman and infrared reflection spectroscopy in black phosphorus. Solid State Commun, 1985, 53: 753-755

111 Xu X L, Song Q J, Wang H F, et al. In-plane anisotropies of polarized Raman response and electrical conductivity in layered tin selenide. ACS Appl Mater Interfaces, 2017, 9: 12601-12607

112 Huang S X, Tatsumi Y, Ling X, et al. In-plane optical anisotropy of layered gallium telluride. ACS Nano, 2016, 10: 8964-8972

113 Ma X L, Guo P J, Yi C J, et al. Raman scattering in the transition-metal dichalcogenides of $1 \mathrm{~T}^{\prime}-\mathrm{MoTe}_{2}, \mathrm{t}_{\mathrm{d}}-\mathrm{MoTe}_{2}$, and $\mathrm{t}_{\mathrm{d}}-\mathrm{WTe}_{2}$. Phys Rev B, 2016, 94: 214105

114 Wolverson D, Crampin S, Kazemi A S, et al. Raman spectra of monolayer, few-layer, and bulk ReSe $e_{2}$ : An anisotropic layered semiconductor. ACS Nano, 2014, 8: 11154-11164

115 Mao N N, Tang J Y, Xie L M, et al. Optical anisotropy of black phosphorus in the visible regime. J Am Chem Soc, 2016, 138: 300-305

116 Kranert C, Sturm C, Schmidt-Grund R, et al. Raman tensor formalism for optically anisotropic crystals. Phys Rev Lett, 2016, 116: 127401

117 Mao N N, Wu J X, Han B W, et al. Birefringence-directed Raman selection rules in 2D black phosphorus crystals. Small, 2016, 12: $2627-2633$

118 Zhang S S, Mao N N, Zhang N, et al. Anomalous polarized Raman scattering and large circular intensity differential in layered triclinic $\mathrm{ReS}_{2}$. ACS Nano, 2017, 11: 10366-10372

119 Wang X S, Xie L M, Zhang J. Preparation, structure and properties of two-dimensional semiconductor alloys (in Chinese). Acta Chim Sin, 2015, 73: 886-894 [王新胜, 谢黎明, 张锦. 二维半导体合金的制备、结构和性质. 化学学报, 2015, 73: 886-894]

120 Mann J, Ma Q, Odenthal P M, et al. 2-Dimensional transition metal dichalcogenides with tunable direct band gaps: $\mathrm{MoS}_{2(1-x)} \mathrm{Se}_{2 x} \mathrm{mono}_{-}$ layers. Adv Mater, 2014, 26: 1399-1404

121 Chen Y F, Wen W, Zhu Y M, et al. Temperature-dependent photoluminescence emission and Raman scattering from $\mathrm{Mo}_{1-x} \mathrm{~W}_{x} \mathrm{~S}_{2} \mathrm{mono}_{-}$ layers. Nanotechnology, 2016, 27: 445705 
122 Feng Q L, Mao N N, Wu J X, et al. Growth of $\mathrm{MoS}_{2(1-x)} \mathrm{Se}_{2 x}(x=0.41-1.00)$ monolayer alloys with controlled morphology by physical vapor deposition. ACS Nano, 2015, 9: 7450-7455

123 Cancado L G, Jorio A, Ferreira E H M, et al. Quantifying defects in graphene via Raman spectroscopy at different excitation energies. Nano Lett, 2011, 11: 3190-3196

124 Eckmann A, Felten A, Mishchenko A, et al. Probing the nature of defects in graphene by Raman spectroscopy. Nano Lett, 2012, 12: 3925-3930 Mignuzzi S, Pollard A J, Bonini N, et al. Effect of disorder on Raman scattering of single-layer MoS $_{2}$. Phys Rev B, 2015, 91 : 195411 Mao N N, Chen Y F, Liu D M, et al. Solvatochromic effect on the photoluminescence of $\mathrm{MoS}_{2}$ monolayers. Small, 2013, 9: 1312-1315 Chakraborty B, Bera A, Muthu D V S, et al. Symmetry-dependent phonon renormalization in monolayer MoS 2 transistor. Phys Rev B, 2012, 85: 161403

128 Ni Z H, Yu T, Lu Y H, et al. Uniaxial strain on graphene: Raman spectroscopy study and band-gap opening. ACS Nano, 2008, 2: $2301-2305$

129 Conley H J, Wang B, Ziegler J I, et al. Bandgap engineering of strained monolayer and bilayer MoS 2 . Nano Lett, 2013, 13: 3626-3630

130 Hui Y Y, Liu X F, Jie W J, et al. Exceptional tunability of band energy in a compressively strained trilayer MoS 2 sheet. ACS Nano, 2013, 7: 7126-7131

131 Wang Y L, Cong C X, Fei R X, et al. Remarkable anisotropic phonon response in uniaxially strained few-layer black phosphorus. Nano Res, 2015, 8: 3944-3953

132 Fei R X, Yang L. Lattice vibrational modes and Raman scattering spectra of strained phosphorene. Appl Phys Lett, 2014, 105: 083120

133 Calizo I, Balandin A A, Bao W, et al. Temperature dependence of the Raman spectra of graphene and graphene multilayers. Nano Lett, 2007, 7: 2645-2649

134 Sahoo S, Gaur A P S, Ahmadi M, et al. Temperature-dependent Raman studies and thermal conductivity of few-layer MoS 2 . J Phys Chem C, 2013, 117: 9042-9047

135 Luo Z, Maassen J, Deng Y X, et al. Anisotropic in-plane thermal conductivity observed in few-layer black phosphorus. Nat Commun, 2015, 6: 8572

136 Liu T H, Chang C C. Anisotropic thermal transport in phosphorene: Effects of crystal orientation. Nanoscale, 2015, 7: 10648-10654

137 Wang X S, Liu H N, Wu J X, et al. Chemical growth of 1T- $\mathrm{TaS}_{2}$ monolayer and thin films: Robust charge density wave transitions and high bolometric responsivity. Adv Mater, 2018, 30: 1800074

138 Zhao R, Wang Y, Deng D, et al. Tuning phase transitions in $1 \mathrm{~T}-\mathrm{TaS}_{2}$ via the substrate. Nano Lett, 2017, 17: 3471-3477

139 Cao Y F, Cai K M, Li L J, et al. Transport and capacitance properties of charge density wave in few-layer $2 \mathrm{H}-\mathrm{TaS}_{2}$ devices. Chin Phys Lett, 2014, 31: 077203

140 Stoltz D, Bielmann M, Schlapbach L, et al. Atomic origin of the scanning tunneling microscopy images of charge-density-waves on 1T-TaSe 2 . Phys B, 2008, 403: 2207-2210

141 Valla T, Fedorov A V, Johnson P D, et al. Quasiparticle spectra, charge-density waves, superconductivity, and electron-phonon coupling in $2 \mathrm{H}-\mathrm{NbSe}_{2}$. Phys Rev Lett, 2004, 92: 086401

142 Mulazzi M, Chainani A, Katayama N, et al. Absence of nesting in the charge-density-wave system 1T-VS ${ }_{2}$ as seen by photoelectron spectroscopy. Phys Rev B, 2010, 82: 2283-2288

$143 \mathrm{Xu} \mathrm{K}$, Chen P Z, Li X L, et al. Ultrathin nanosheets of vanadium diselenide: A metallic two-dimensional material with ferromagnetic charge-density-wave behavior. Angew Chem Int Ed, 2013, 52: 10477-10481

144 He R, Okamoto J, Ye Z P, et al. Distinct surface and bulk charge density waves in ultrathin 1T- TaS 2 . Phys Rev B, 2016, $94: 201108$

$145 \mathrm{Fu} \mathrm{W}$, Chen Y, Lin J H, et al. Controlled synthesis of atomically thin $1 \mathrm{~T}-\mathrm{TaS}_{2}$ for tunable charge density wave phase transitions. Chem Mater, 2016, 28: 7613-7618

146 Albertini O R, Zhao R, McCann R L, et al. Zone-center phonons of bulk, few-layer, and monolayer 1T-TaS ${ }_{2}$ : Detection of commensurate charge density wave phase through Raman scattering. Phys Rev B, 2016, 93: 214109

147 Hossain M, Wu J X, Wen W, et al. Chemical vapor deposition of 2D vanadium disulfide and diselenide and Raman characterization of the phase transitions. Adv Mater Interfaces, 2018, 5: 1800528 
Summary for “二维材料的拉曼光谱研究进展”

\title{
Progress on Raman spectroscopy of two-dimensional materials
}

\author{
Juanxia $\mathrm{Wu}^{1,2,3}$ \& Liming Xie $\mathrm{X}^{1,2,3^{*}}$ \\ ${ }^{1}$ Key Laboratory of Standardization and Measurement for Nanotechnology, National Center for Nanoscience and Technology, Chinese Academy of \\ Sciences, Beijing 100190, China; \\ ${ }^{2}$ Center for Excellence in Nanoscience, National Center for Nanoscience and Technology, Chinese Academy of Sciences, Beijing 100190, China; \\ ${ }^{3}$ School of Nanoscience and Technology, International College, University of Chinese Academy of Sciences, Beijing 100049, China \\ * Corresponding author, E-mail: xielm@nanoctr.cn
}

Two-dimensional (2D) materials have attracted intense interest due to the planar structure, unique properties and broad applications. Raman spectroscopy is a quick and nondestructive finger-print method in the material science and has been used to study the material properties for many years. It plays a very important role in the structural characterization of 2D materials, which can be used to probe the electronic band structure, phonon dispersion and electron-phonon interaction in 2D materials. This review summarizes the recent progress of structural studying of $2 \mathrm{D}$ materials using Raman spectroscopy. Firstly, the typical Raman features of 2D materials are introduced on the basis of systematically analyzing the structures and Raman selection rules. Secondly, Raman spectroscopy can be used to quantify the structural parameters of 2D materials. The electronic band structure of 2D materials can be modified due to the interlayer interaction as the thickness increases, leading to the change of typical Raman features of 2D materials. Therefore, according to the position, width and intensity of Raman peaks, the layer number of 2D materials can be quickly and precisely distinguished. At the edge of 2D materials, breakdown of the Raman selection rules caused by the decrease of structural symmetry results in the anomalous Raman signals (additional phonon modes), and then the edge chirality of 2D materials can be determined. For 2D materials with in-plane anisotropic structure, the anisotropic polarized Raman intensity can be used to identify the crystalline orientation 2D materials. The change of composition in 2D alloy materials can induce the shift of Raman peaks. Combining with the MREI model analysis, the alloy composition quantification of unknown 2D alloy materials and the force parameters of atomic vibration can be both obtained using Raman spectroscopy. Thirdly, external environment, i.e. defect, doping, strain, and thermal effect, will strongly modify the electronic band structure, and then affect the Raman signals. Existence of defects can lead to the new Raman vibrational modes in 2D materials, so the defect density can be monitored according to the intensity ratio of this new Raman peak and the typical Raman features in the 2D materials. Based on the shift of Raman peaks and influence of doping, strain, and temperature on the electronic structure of 2D materials, we also investigate the doping density, strain, and the thermal conductivity of 2D materials. At last, the change of structure during the phase transition of $2 \mathrm{D}$ charge density wave materials can also induce the change of Raman selection rules, so the Raman spectroscopy has been applied to on the research of phase transition characteristics according to the change of Raman features.

two-dimensional materials, Raman spectroscopy, structural characterization, Raman selection rule

doi: 10.1360/N972018-00849 